Pure and Applied Mathematics Quarterly

Volume 1, Number 4

(Special Issue: In Memory of

Armand Borel, Part 3 of 3 )

$827-850,2005$

\title{
Special Skew Shapes and Type $A$ Affine Hecke Algebras
}

\author{
Jun $\mathrm{Hu}$
}

\begin{abstract}
Let $l \in \mathbb{N}, l \geq m \in \mathbb{N}$. In this paper, we introduce the notions of $l$-special skew shapes (resp. $(l, m)$-special skew shapes) and study their properties. Applications of these objects in the representation theory of type $A$ affine Hecke algebras $\mathcal{H}_{n}^{\text {aff }}$ with parameter $q \in K$ are given, where $q=\sqrt[l]{1}$; or $q=1$ and char $K=l$. Using Young's seminormal construction, we show that, for each $l$-special skew shape $\lambda / \mu$ with $\operatorname{Std}_{l}(\lambda, \mu) \neq \emptyset$ (see Section 1 for definition of $\operatorname{Std}_{l}(\lambda, \mu)$ ), there is an indecomposable representation of $\mathcal{H}_{n}^{\text {aff }}$; while for each $(l, m)$-special skew shape, there is an irreducible representation of $\mathcal{H}_{n}^{\text {aff }}$. Our notion of $(l, m)$-special skew shape generalizes the notion of $(l, m)$-special partition in earlier work of O. Mathieu (see $[\mathrm{M}]$ ) and of $\mathrm{H}$. Wenzl (see $[\mathrm{W}])$.
\end{abstract}

\section{INTRODUCTION}

Let $n$ be a positive integer. Let $\mathfrak{S}_{n}$ be the symmetric group on $n$ letters. Let $K$ be a field and $q \in K^{\times}$. The Iwahori-Hecke algebra $\mathcal{H}_{q}\left(\mathfrak{S}_{n}\right)$ of $\mathfrak{S}_{n}$ is the unital $K$-algebra with generators $T_{1}, \cdots, T_{n-1}$ and relations

Received March 25, 2005.

2000 Mathematics Subject Classification. 16G99

Research supported by Alexander von Humboldt Foundation and National Natural Science Foundation of China(Project 10401005) and partly supported by Program NCET and the URF of Victoria University of Wellington. The author wish to thank Professor Richard Dipper for several helpful discussions. He also wishes to thank the referee for careful reading and helpful comments. 


$$
\begin{aligned}
& \left(T_{i}+1\right)\left(T_{i}-q\right)=0, \quad \text { for } 1 \leq i<n, \\
& T_{i} T_{i+1} T_{i}=T_{i+1} T_{i} T_{i+1}, \quad \text { for } 1 \leq i<n-1, \\
& T_{i} T_{j}=T_{j} T_{i}, \quad \text { for } 1 \leq i<j-1<n-1 .
\end{aligned}
$$

Note that if $q=1$, one recovers the group algebra of $\mathfrak{S}_{n}$ over $K$.

Let $l$ be the smallest positive integer such that $1+q+q^{2}+\cdots+q^{l-1}=0$ in $K ; l=\infty$ if no such integer exists. It is well-known that $\mathcal{H}_{q}\left(\mathfrak{S}_{n}\right)$ is (split) semi-simple if and only if $l>n$. Much more interesting is the case when $l \leq n$, i.e., the modular case. For each partition $\lambda=\left(\lambda_{1}, \lambda_{2}, \cdots\right)$ of $n$, there is a Specht module $S^{\lambda}$ for $\mathcal{H}_{q}\left(\mathfrak{S}_{n}\right)$. If $l>n$, then $S^{\lambda}$ is irreducible, and as $\lambda$ varies over the partitions of $n$, the Specht module varies over the pairwise non-isomorphic (absolutely) irreducible modules for $\mathcal{H}_{q}\left(\mathfrak{S}_{n}\right)$. In general, $S^{\lambda}$ is not necessarily irreducible, but the dimension of $S^{\lambda}$ is independent of $K$ and could be given by an explicit combinatorial formula depending only on $\lambda$. If $\lambda$ is $l$-restricted, i.e., $\lambda_{i}-\lambda_{i+1}<l$ for any $i$, then $S^{\lambda}$ has a unique top composition factor $D^{\lambda}$, and as $\lambda$ varies over the $l$-restricted partitions of $n$, the $D^{\lambda}$ varies over the pairwise non-isomorphic (absolutely) irreducible modules for $\mathcal{H}_{q}\left(\mathfrak{S}_{n}\right)$.

There are two most important outstanding problems in modular representation theory for $\mathcal{H}_{q}\left(\mathfrak{S}_{n}\right)$, namely,

(i) determine the multiplicities of each irreducible module in a given Specht module;

(ii) determine the dimension of each irreducible module, or more generally, of each indecomposable module.

If $q \neq 1$ and $K$ has characteristic 0 , then the work of Lascoux-Leclerc-Thibon and Ariki yields a complete solution to Problem (i), using the theory of canonical bases for quantum groups (see $[\mathrm{A}]$ and [LLT]). In general (still $q \neq 1$ ), there is a James' Conjecture (see [J]); while Problem (ii) remains open. To the best of our knowledge, there is not even a conjectured formula.

In $[\mathrm{M}]$, a partial solution to Problem (ii) in the case where $q=1$ was found by O. Mathieu, who computed the dimension of some irreducible representations of $\mathfrak{S}_{n}$ in characteristic $l>0$ (in particular, here $l$ is a prime number). The representations considered in his paper are associated with partitions $\lambda=\left(\lambda_{1}, \lambda_{2}, \cdots\right)$ such that $\ell(\lambda) \leq m$ and $\lambda_{1}-\lambda_{m} \leq l-m$ for a given positive integer $m$ (called $(l, m)$-special partition). His formula is based on Schur-Weyl duality and a variant of Verlinde's formula which computes some tensor product multiplicities of indecomposable tilting modules in $n$-tensor space. In general those tensor product multiplicities are unknown. In [W], in his study of Hecke algebras $\mathcal{H}_{q}\left(\mathfrak{S}_{n}\right)$ at 
a primitive $l$-th root of unity, $\mathrm{H}$. Wenzl also considered Hecke modules parameterized by partitions satisfying the same condition.

The modules considered by Mathieu are in fact the same ${ }^{1}$ as the modules considered by Wenzl. This is the motivation of the present paper. We generalize the notion of $(l, m)$-special partition to the notion of $(l, m)$-special skew shape. Furthermore, we propose the notion of $l$-special skew shape, which is new even in the case of partition. Note that the representation of type $A$ Iwahori-Hecke algebra is a part of the representation of the type $A$ affine Hecke algebra. It turns out that our new combinatorial objects have applications in the representation theory of type $A$ affine Hecke algebras.

We use Young's seminormal construction (see [Ho], [AK], [HaR], [R1-4]) in these applications ${ }^{2}$. We obtain a family of indecomposable modules as well as a family of irreducible modules. Note that Ram ([R4]) developed calibrated irreducible representation theory for affine Hecke algebras of general type. The irreducible modules we obtained are the irreducible calibrated representations (in type $A$ ) obtained by Ram. But we do not know if every irreducible calibrated representation in type $A$ is isomorphic to one of the irreducible modules we obtained. We index those irreducible modules by $(l, m)$-special skew shape, while Ram indexed irreducible calibrated representations by skew local regions. Note that when $l=\infty$, the skew local regions (in the case of type $A$ ) correspond exactly to usual skew shapes. In general, they correspond to certain $l$-periodic configuration of boxes. ${ }^{3}$

To state the main result of this paper, we need some combinatorial notations. Let $n$ be a positive integer. Let $K, q, l$ be defined as before. A partition of $n$ is a sequence $\lambda=\left(\lambda_{1}, \lambda_{2}, \cdots\right)$ of non-negative integers such that $\lambda_{1} \geq \lambda_{2} \geq \cdots$ and $n=|\lambda|:=\sum_{i \geq 1} \lambda_{i}$. If $\lambda$ is a partition of $n$, we write $\lambda \vdash n$ and denote by $\ell(\lambda)$ the largest integer $j$ such that $\lambda_{j} \neq 0$. The Young diagram of $\lambda$ is defined as $[\lambda]:=\left\{(a, b) \mid 1 \leq b \leq \lambda_{a}\right\}$. The elements of $[\lambda]$ are called nodes. We shall often identify a partition with its Young diagram. If $\lambda$ and $\mu$ are partitions such that $\mu_{i} \leq \lambda_{i}$ for all $i$ we write $\mu \subseteq \lambda$, in that case we use $\lambda / \mu$ to denote the diagram which consists of all nodes of $[\lambda]$ which are not in $[\mu]$, and we call $\nu=\lambda / \mu$ a skew shape.

\footnotetext{
$1_{\text {This seems to be known to some experts. But since we can not find suitable references }}$ anywhere, a proof for this will be given (Theorem 1.3).

${ }^{2}$ For applications of Young's seminormal construction in modular representations of cyclotomic Hecke algebras, see [Hu1] and [Hu2].

${ }^{3}$ As the referee said, if a skew shape is $(l, m)$-special then one can stick two copies of it together such that the two copies differ by $m$ rows and $l$ diagonals, the resulting shape is skew again. Repeating this process one obtains a skew local region. On the other hand if a skew local region is given, such that the shape is repeated every $m$ rows upwards, one get an $(l, m)$-special skew shape taking $m$ consecutive rows of the configuration.
} 
A partition $\lambda=\left(\lambda_{1}, \lambda_{2}, \cdots\right)$ is called an $l$-special partition if $\ell(\lambda) \leq l$ and $\lambda_{1}-\lambda_{\ell(\lambda)} \leq l-\ell(\lambda)$. Let $\mu$ be an $l$-special partition. A skew shape $\nu$ is called an $l$-special skew shape with respect to $\mu$ if $\nu=\lambda / \mu$ for some $l$-special partition $\lambda$. For each $l$-special skew shape $\nu=\lambda / \mu$, we denote by $\operatorname{Std}_{l}(\lambda, \mu)$ the set of all the $l$-special standard $\nu$-tableaux with respect to $\mu$ (see 2.8 for definitions of $l$ special standard tableaux). After proving a number of properties about $l$-special standard $\nu$-tableaux, we get the first main result of this paper.

Theorem 1.1. Let $\nu=\lambda / \mu$ be an l-special skew shape with respect to $\mu$. Suppose that $[\nu]$ contains $n$ nodes. If $\operatorname{Std}_{l}(\lambda, \mu) \neq \emptyset$, then there is an indecomposable representation $I(\lambda, \mu)$ of $\mathcal{H}_{n}^{\text {aff }}$ such that $\operatorname{dim} I(\lambda, \mu)$ is equal to $\# \operatorname{Std}_{l}(\lambda, \mu)$, i.e., the number of all the l-special standard $\nu$-tableaux with respect to $\mu$.

Actually we shall construct $I(\lambda, \mu)$ with a basis labelled by all the $l$-special standard $\nu$-tableaux with respect to $\mu$, and the action of each generator of $\mathcal{H}_{n}^{\text {aff }}$ on such a basis is defined by the standard Young's seminormal construction.

Let $m$ be a fixed positive integer with $m \leq l$. A partition $\lambda$ is called an $(l, m)$-special partition if $\ell(\lambda) \leq m$ and $\lambda_{1}-\lambda_{m} \leq l-m$. Let $\mu$ be an $(l, m)$ special partition. A skew shape $\nu$ is called an $(l, m)$-special skew shape with respect to $\mu$ if $\nu=\lambda / \mu$ for some $(l, m)$-special partition $\lambda$. For each $(l, m)$-special skew shape $\nu=\lambda / \mu$, we denote by $\operatorname{Std}_{(l, m)}(\lambda, \mu)$ the set of all the $(l, m)$-special standard $\nu$-tableaux with respect to $\mu$ (see 2.9 for definitions of $(l, m)$-special standard tableaux). After proving a number of properties about $(l, m)$-special standard $\nu$-tableaux, we get the second main result of this paper.

Theorem 1.2. Let $\nu=\lambda / \mu$ be an $(l, m)$-special skew shape with respect to $\mu$. Suppose that $[\nu]$ contains $n$ nodes. Then $\operatorname{Std}_{(l, m)}(\lambda, \mu) \neq \emptyset$, and there is an irreducible representation $D(\lambda, \mu)$ of $\mathcal{H}_{n}^{\text {aff }}$ such that $\operatorname{dim} D(\lambda, \mu)$ is equal to $\# \operatorname{Std}_{(l, m)}(\lambda, \mu)$, i.e., the number of $(l, m)$-special standard $\nu$-tableaux with respect to $\mu$.

Actually we shall construct $D(\lambda, \mu)$ with a basis labelled by all the $(l, m)$-special standard $\nu$-tableaux with respect to $\mu$, and the action of each generator of $\mathcal{H}_{n}^{\text {aff }}$ on such a basis is also defined by the standard Young's seminormal construction.

Note that our $(l, m)$-special skew shapes with respect to $\emptyset$ are just those partitions considered in $[\mathrm{M}]$ and $[\mathrm{W}]$. For each $(l, m)$-special partition (i.e., $(l, m)$ special skew shapes with respect to $\emptyset) \lambda$, we write $D(\lambda)$ instead of $D(\lambda, \emptyset)$. Let $D^{\lambda}$ be the irreducible $\mathcal{H}_{q}\left(\mathfrak{S}_{n}\right)$-module, which is the unique top irreducible composition factor of the Specht module $S^{\lambda}$. We have that

Theorem 1.3. For each $(l, m)$-special partition $\lambda$, we have that $D(\lambda) \cong D^{\lambda}$. 
In particular, this proves that the irreducible modules $D(\lambda)=D(\lambda, \emptyset)$ we studied here coincide with those studied in $[\mathrm{M}]$, hence in that case Theorem 1.2 reduces to the result of $[\mathrm{M}]$ and of $[\mathrm{W}]$.

\section{Preliminaries}

Let $n$ be a positive integer. The symmetric group $\mathfrak{S}_{n}$ acts on $\{1,2, \cdots, n\}$ from the right. For each $1 \leq i<n$, let $s_{i}:=(i, i+1)$. Then $s_{1}, \cdots, s_{n-1}$ are the standard Coxeter generators of $\mathfrak{S}_{n}$. Let $w \in \mathfrak{S}_{n}$. A word $w=s_{i_{1}} \cdots s_{i_{k}}$ for $w$ is a reduced expression for $w$ if $k$ is minimal; in this case we say that $w$ has length $k$ and write $\ell(w)=k$.

Let $K$ be a field and let $q$ be an invertible element in $K$. Hereafter we shall assume that $l$ is the smallest positive integer such that $1+q+q^{2}+\cdots+q^{l-1}=0$ in $K$. If $q=1$, this amounts to say that the field $K$ has characteristic $l>0$. Let $\mathcal{H}_{q}\left(\mathfrak{S}_{n}\right)$ be the Hecke algebra of $\mathfrak{S}_{n}$ as defined in Section 1. If $q=1$, we shall use the generator $s_{i}$ instead of $T_{i}$ for $1 \leq i<n$.

Definition 2.1. The affine Hecke algebra $\mathcal{H}_{n}^{\text {aff }}$ is the $K$-algebra, which as a $K$-linear space is isomorphic to

$$
\mathcal{H}_{q}\left(\mathfrak{S}_{n}\right) \otimes_{K} K\left[X_{1}^{ \pm 1}, \cdots, X_{n}^{ \pm 1}\right] .
$$

The algebra structure is given by requiring that $\mathcal{H}_{q}\left(\mathfrak{S}_{n}\right)$ and $K\left[X_{1}^{ \pm 1}, \cdots, X_{n}^{ \pm 1}\right]$ are subalgebras and that

$$
T_{i} f-{ }^{s_{i}} f T_{i}=(q-1) \frac{f-{ }^{s_{i}} f}{1-X_{i} X_{i+1}^{-1}}, \quad \forall f \in K\left[X_{1}^{ \pm 1}, \cdots, X_{n}^{ \pm 1}\right],
$$

if $q \neq 1$; or

$$
s_{i} f-{ }^{s_{i}} s_{i}=\frac{f-{ }^{s_{i}} f}{X_{i+1}-X_{i}}, \quad \forall f \in K\left[X_{1}^{ \pm 1}, \cdots, X_{n}^{ \pm 1}\right],
$$

if $q=1$. Here $s_{i} \in \mathfrak{S}_{n}$ acts on $K\left[X_{1}^{ \pm 1}, \cdots, X_{n}^{ \pm 1}\right]$ by permuting $X_{i}$ and $X_{i+1}$.

Note that the relation (2.2) is equivalent to

$$
\begin{aligned}
T_{i} X_{i} T_{i} & =q X_{i+1}, \quad \forall i \text { with } 1 \leq i<n, \\
T_{i} X_{j} & =X_{j} T_{i}, \quad \forall j \notin\{i, i+1\},
\end{aligned}
$$

while the relation $(2.3)$ is equivalent to

$$
\begin{aligned}
& X_{i+1}=s_{i} X_{i} s_{i}+s_{i}, \quad \forall i \text { with } 1 \leq i<n, \\
& s_{i} X_{j}=X_{j} s_{i}, \quad \forall j \notin\{i, i+1\} .
\end{aligned}
$$

Let $a$ be a positive integer and let $\lambda$ be a partition of $a$. A $\lambda$-tableau $\mathfrak{t}$ is obtained from the Young diagram $[\lambda]$ by replacing each node by one of the integers $1,2, \cdots, a$, allowing no repeats. We write Shape $(\mathfrak{t})=\lambda$. $\mathfrak{t}$ is standard if the entries 
increase from left to right in each row and from top to bottom down each column. Let $\mathfrak{t}$ be a standard $\lambda$-tableau. For any integer $i$ with $1 \leq i \leq a$, we denote by $\mathfrak{t} \downarrow_{i}$ the tableau obtained by removing all nodes with entry greater than $i$. It is easy to see that Shape $\left(\mathfrak{t} \downarrow_{i}\right)$ is again a partition.

A node $\gamma$ on the boundary of $[\lambda]$ is called a removable node if $[\lambda]-\{\gamma\}$ is again a Young diagram of a partition, in that case $\gamma$ is also called an addable node of $[\lambda]-\{\gamma\}$. For any partition $\mu$, we write $\mu \rightarrow \lambda$ if $[\lambda]=[\mu] \cup\{\gamma\}$ for some removable node $\gamma \in[\lambda]$. Let $\lambda$ be a partition of $n$. We define a standard $\lambda$-chain to be a chain $C=(C(1) \rightarrow C(2) \rightarrow \cdots \rightarrow C(n))$ of partitions such that $C(i) \vdash i$ for each $i$ and $C(n)=\lambda$.

Lemma 2.6 For any partition $\lambda$ of $n$, the map which sends each standard $\lambda$-tableau $\mathfrak{t}$ to the standard $\lambda$-chain

$$
\left(\operatorname{Shape}\left(\mathfrak{t} \downarrow_{1}\right) \rightarrow \text { Shape }\left(\mathfrak{t} \downarrow_{2}\right) \rightarrow \cdots \rightarrow \text { Shape }\left(\mathfrak{t} \downarrow_{n}\right)=\lambda\right)
$$

defines a bijection between the set of standard $\lambda$-tableaux and the set of standard $\lambda$-chains.

The above result allows a generalization in the following sense. For any two partitions $\lambda$, $\mu$ with $\mu \subseteq \lambda$. Let $n=|\lambda|-|\mu|$. A chain $C=(C(0) \rightarrow C(1) \rightarrow$ $\cdots \rightarrow C(n))$ of partitions such that $C(i) \vdash(|\mu|+i)$ for each $i, C(0)=\mu$ and $C(n)=\lambda$, will be called a standard $\lambda$-chain starting from $\mu$. Recall our definition of skew shape in Section 1. Let $\nu=\lambda / \mu$ be a skew shape with $n$ nodes, where $\mu \vdash k$ and $\lambda \vdash(n+k)$. A standard $\nu$-tableau is a filling of the nodes in the skew shape $\nu$ with the numbers $1,2, \cdots, n$ such that the numbers increase from left to right in each row and from top to bottom down each column. Let $\mathfrak{t}$ be a standard $\nu$-tableau. For any integer $i$ with $1 \leq i \leq n$, we denote by $\mathfrak{t} \downarrow_{i}$ the tableau obtained by removing all nodes with entry greater than $i$. It is easy to see that Shape $\left(\mathfrak{t} \downarrow_{i}\right)$ is again a skew shape.

Lemma 2.7 With the above notations, the map which sends each standard $\nu$-tableau $\mathfrak{t}$ to the chain

$$
\left([\mu] \rightarrow[\mu] \cup \operatorname{Shape}\left(\mathfrak{t} \downarrow_{1}\right) \rightarrow[\mu] \cup \operatorname{Shape}\left(\mathfrak{t} \downarrow_{2}\right) \rightarrow \cdots \rightarrow[\mu] \cup \operatorname{Shape}\left(\mathfrak{t} \downarrow_{n}\right)=[\lambda]\right)
$$

defines a bijection between the set of standard $\nu$-tableaux and the set of standard $\lambda$-chains starting from $\mu$.

Recall our definition of $l$-special partitions (resp. $l$-special skew shape) in Section 1.

Definition 2.8 Let $\mu$ be an l-special partition. Let $\nu=\lambda / \mu$ be an l-special skew shape with respect to $\mu$, where $\mu \vdash k$ and $\lambda \vdash(n+k)$ for some positive integers $k, n$. A standard $\nu$-tableau $\mathfrak{t}$ is called an $l$-special standard $\nu$-tableau with respect to $\mu$ if for each $1 \leq j \leq n,[\mu] \cup$ Shape $\left(\mathfrak{t} \downarrow_{j}\right)$ is an l-special partition. 
Recall also our definition of $(l, m)$-special partitions (resp. $(l, m)$-special skew shape) in Section 1.

Definition 2.9 Let $\mu$ be an $(l, m)$-special partition. Let $\nu=\lambda / \mu$ be an $(l, m)$-special skew shape with respect to $\mu$, where $\mu \vdash k$ and $\lambda \vdash(n+k)$ for some positive integers $k, n$. A standard $\nu$-tableau $\mathfrak{t}$ is called an $(l, m)$-special standard $\nu$-tableau with respect to $\mu$ if for each $1 \leq j \leq n$, $[\mu] \cup \operatorname{Shape}\left(\mathfrak{t} \downarrow_{j}\right)$ is an $(l, m)$-special partition.

Example 2.10 Suppose that $n=5, m=5, l=8$, and $\lambda=\left(4,3^{2}, 1^{2}\right), \mu=$ $\left(3,2,1^{2}\right)$, Then $\nu:=\lambda / \mu$ is an $(8,5)$-special skew shape with respect to $\mu$.

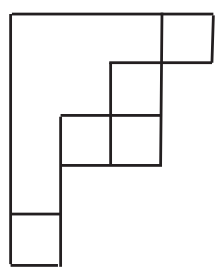

In this case, among the following two standard $\nu$-tableaux,
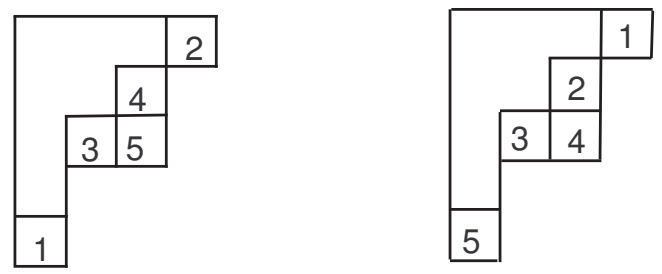

the first one is an $(8,5)$-special standard $\nu$-tableau with respect to $\mu$, while the second one is not an $(8,5)$-special standard $\nu$-tableau with respect to $\mu$.

Remark 2.11 1) By definition, an $l$-special partition $\lambda$ is automatically an $(l, \ell(\lambda))$-special partition.

2) Let $m \leq l$ be two fixed positive integers. For any partition $\lambda$ with $\ell(\lambda) \leq m$, $\lambda$ is an $(l, m)$-special partition only if $\lambda$ is an $l$-special partition.

3) Let $m \leq l$ be two fixed positive integers. Let $\nu=\lambda / \mu$ be an $(l, m)$-special skew shape with respect to $\mu$ and $\mathfrak{t}$ be a standard $\nu$-tableau. Then $\mathfrak{t}$ is $(l, m)$ special standard with respect to $\mu$ only if $\mathfrak{t}$ is an $l$-special standard with respect to $\mu$.

Let $\nu$ be a skew shape. Among all the partition $\lambda$ such that $\nu=\lambda / \mu$ for some partition $\mu$, there is a unique minimal one (under the dominance order $\unlhd$ ) which will be denoted by $\lambda_{\nu}$, then $\nu=\lambda_{\nu} / \mu_{\nu}$, for some partition $\mu_{\nu}$ (which is 
also uniquely determined by $\nu$ ), and $\lambda_{\nu} \subseteq \lambda, \mu_{\nu} \subseteq \mu$. In particular, both the first row and the first column of $\left[\lambda_{\nu}\right]$ contain nodes of $\nu$. Note that our definitions of $l$-special (resp. $(l, m)$-special) skew shapes and standard tableaux depend on a first choice of $\mu$. However, they are compatible with inclusion relations. That is, if $\nu=\widetilde{\lambda} / \widetilde{\mu}=\lambda / \mu$ is a skew shape such that $\lambda \subseteq \widetilde{\lambda}, \mu \subseteq \widetilde{\mu}$, then $l$-special (resp. $(l, m)$-special) skew shapes and standard tableaux with respect to $\widetilde{\mu}$ remain $l$ special (resp. $(l, m)$-special) with respect to $\mu$. As a result, if $\nu=\lambda / \mu$ is an $l$-special skew shape with respect to $\mu$ and $\mathfrak{t}$ is an $l$-special standard tableau with respect to $\mu$, for each $1 \leq j \leq n$, let $\nu(j):=\operatorname{Shape}\left(\mathfrak{t} \downarrow_{j}\right)$, then $\nu(j)$ is an $l$-special skew shape with respect to $\mu_{\nu(j)}$, and $\mathfrak{t} \downarrow_{j}$ is an $l$-special standard $\nu(j)$ tableau with respect to $\mu_{\nu(j)}$. The same is true for $(l, m)$-special skew shapes and $(l, m)$-special standard tableaux. To simplify notations, hereafter, we make the following conventions: whenever we write " $\lambda / \mu$ is l-special (resp. $(l, m)$-special), or an l-special (resp. $(l, m)$-special) skew shape $\lambda / \mu$, or an l-special (resp. $(l, m)$ special) standard $\lambda / \mu$-tableau", we always mean that they are defined with respect to $\mu$.

Example 2.12 Suppose that $n=6, l=8$, and $\lambda=\left(6,4^{2}, 2\right), \mu=(5,3,2)$, Then $\nu:=\lambda / \mu$ is an 8 -special skew shape but not an $(8, m)$-special skew shape whenever $m \geq 5$.

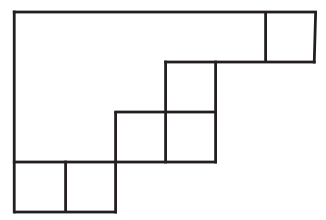

In this case, among the following two standard $\nu$-tableaux,
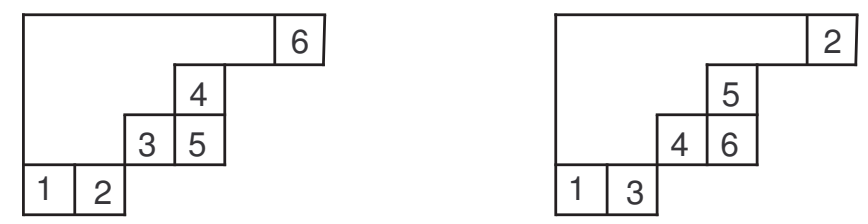

the first one is an 8-special standard $\nu$-tableau with respect to $\mu$, while the second one is not an 8-special standard $\nu$-tableau with respect to $\mu$.

Remark 2.13 For any $(l, m)$-special skew shape $\nu=\lambda / \mu$ with $n$ nodes, let $\mathfrak{t}_{\nu}$ be the standard $\nu$-tableau in which the number $1,2, \cdots, n$ appear in order (i.e., from top to bottom) along successive columns. It is easy to see that $\mathfrak{t}_{\nu}$ is an $(l, m)$-special standard $\lambda / \mu$-tableau. In particular, $\operatorname{Std}_{(l, m)}(\lambda, \mu) \neq \emptyset$. However, for an $l$-special skew shape $\nu=\lambda / \mu$, it may happen that $\operatorname{Std}_{l}(\lambda, \mu)=\emptyset$. For example, if $n=8, l=5, \nu:=(8,5) /(5)$. Then $\operatorname{Std}_{5}((8,5),(5))=\emptyset$. 
Let $\lambda$ be a partition of $n$. If $\gamma=(a, b)$ and $\gamma^{\prime}=\left(a^{\prime}, b^{\prime}\right)$ are two nodes of $[\lambda]$, we say that $\gamma$ is below $\gamma^{\prime}$, or $\gamma^{\prime}$ is above $\gamma$ if $a>a^{\prime}$. For any $\gamma=(a, b) \in[\lambda]$, we define $\operatorname{Res}(\gamma):=b-a \in \mathbb{Z}$. For any skew shape $\nu=\lambda / \mu$, we define (for each $\gamma \in[\nu]$ ) $\operatorname{Res}(\gamma)$ by regarding $\gamma$ as a node in $[\lambda]$. Note that our definition of the Young diagram $[\mu]$ already enable us to identify $[\mu]$ with a subset of $\mathbb{Z}^{2}$, or equivalently, to fix a coordinate plane. By parallel translation, one can get new identification and hence new coordinate plane. Therefore one can define new Res function. However, the difference between the Res values of any two nodes in $[\nu]$ does not change in the new coordinate plane obtained by parallel translation. Note that the second condition for $\lambda$ being $l$-special (resp., $(l, m)$-special) is equivalent to $\operatorname{Res}\left(\gamma_{1}\right)-\operatorname{Res}\left(\gamma_{2}\right) \leq l-1$, where $\gamma_{1}=\left(1, \lambda_{1}\right), \gamma_{2}=\left(\ell(\lambda), \lambda_{\ell(\lambda)}\right)$ (resp., $\gamma_{2}=$ $\left.\left(m, \lambda_{m}\right)\right)$. This value is the axial distance of these two nodes. Removing one node from a special partition produces a non-special partition only if the axial distance is equal to $l-1$ and the removed node is $\gamma_{2}$ (see Lemma 2.17 and Lemma 2.19). ${ }^{4}$ For any standard $\nu$-tableau $\mathfrak{t}$, and for any integer $k$ with $1 \leq k \leq n$, we use $\operatorname{Res}_{\mathfrak{t}}(k)$ to denote $\operatorname{Res}(\gamma)$ if $\gamma \in[\nu]$ is occupied by $k$ in $\mathfrak{t}$.

Lemma 2.14 Let $\lambda$ be an $l$-special partition with $n$ nodes. Let $\gamma \neq \gamma^{\prime}$ be two removable nodes of $[\lambda]$. Then we have that

$$
0<\left|\operatorname{Res}(\gamma)-\operatorname{Res}\left(\gamma^{\prime}\right)\right|<l .
$$

Moreover, the same is true if one replaces " $l$-special" by " $(l, m)$-special".

Proof. Suppose that $\gamma=(a, b), \gamma^{\prime}=\left(a^{\prime}, b^{\prime}\right)$. Without loss of generality we can assume that $\gamma$ is above $\gamma^{\prime}$, then

$$
1 \leq a<a^{\prime} \leq \ell(\lambda), \quad \lambda_{\ell(\lambda)} \leq b^{\prime}<b \leq \lambda_{1},
$$

and (as $\lambda$ is $l$-special) $\lambda_{1}-\lambda_{\ell(\lambda)} \leq l-\ell(\lambda)$. Hence

$$
\begin{aligned}
1<\operatorname{Res}(\gamma)-\operatorname{Res}\left(\gamma^{\prime}\right) & =(b-a)-\left(b^{\prime}-a^{\prime}\right) \leq \lambda_{1}-\lambda_{\ell(\lambda)}+\ell(\lambda)-1 \\
& \leq l-1
\end{aligned}
$$

as required. By remark 2.11, it is easy to see that the same is true if one replaces "l-special" by " $(l, m)$-special".

Lemma 2.15 Let $\nu=\lambda / \mu$ be an $l$-special skew shape with $n$ nodes. Let $\mathfrak{t}$ be an $l$-special standard $\lambda / \mu$-tableau. For any integers $k$ with $1 \leq k<n$, we have that

$$
0<\left|\operatorname{Res}_{\mathfrak{t}}(k)-\operatorname{Res}_{\mathfrak{t}}(k+1)\right|<l,
$$

and $\left|\operatorname{Res}_{\mathfrak{t}}(k)-\operatorname{Res}_{\mathfrak{t}}(k+1)\right|=1$ if and only if $k, k+1$ lie in the same row or the same column of $\mathfrak{t}$. Moreover, the same is true if one replaces "l-special" by "(l,m)-special".

\footnotetext{
${ }^{4}$ We thank the referee for pointing out this.
} 
Proof. Let $\gamma=(a, b) \in[\lambda]$ be the node occupied by $k$ in $\mathfrak{t}$, and let $\gamma^{\prime}=$ $\left(a^{\prime}, b^{\prime}\right) \in[\lambda]$ be the node occupied by $k+1$ in $\mathfrak{t}$. Let $[\widetilde{\mu}]:=[\mu] \cup$ Shape $\left(\mathfrak{t} \downarrow_{k+1}\right)$. By definition, $\widetilde{\mu}$ is an $l$-special partition and $\gamma^{\prime}$ is a removable node of $\widetilde{\mu}$.

If $\gamma, \gamma^{\prime}$ lie in the same row or the same column of $\lambda$, the conclusion is clear (as $l>1$ ); otherwise $\gamma$ would also be a removable node of $\widetilde{\mu}$. Then we can apply Lemma 2.14, and the lemma follows.

For any skew shape $\nu=\lambda / \mu$ (with $n$ nodes) and any node $\gamma \in[\nu]$, we define

$$
\operatorname{res}(\gamma):=\operatorname{Res}(\gamma)+l \mathbb{Z} \in \mathbb{Z} / l \mathbb{Z}
$$

For any standard $\nu$-tableau $\mathfrak{t}$ and any integer $k$ with $1 \leq k \leq n$, we define $\operatorname{res}_{\mathfrak{t}}(k):=\operatorname{res}(\gamma)$ if $\gamma$ is occupied by $k$ in $\mathfrak{t}$.

Lemma 2.16 Let $\nu=\lambda / \mu$ be an $l$-special skew shape. For any $l$-special standard $\lambda / \mu$-tableaux $\mathfrak{s}, \mathfrak{t}$, if $\operatorname{res}_{\mathfrak{s}}(k)=\operatorname{res}_{\mathfrak{t}}(k), \forall 1 \leq k \leq n$, then $\mathfrak{s}=\mathfrak{t}$. Moreover, the same is true if one replaces " $l$-special" by " $(l, m)$-special".

Proof. Let $\gamma=(a, b) \in[\lambda]$ be the node occupied by 1 in $\mathfrak{s}$, and let $\gamma^{\prime}=\left(a^{\prime}, b^{\prime}\right) \in$ $[\lambda]$ be the node occupied by 1 in $\mathfrak{t}$. Then both $\gamma$ and $\gamma^{\prime}$ are addable nodes of $\mu$. By assumption, $\operatorname{res}(\gamma)=\operatorname{res}\left(\gamma^{\prime}\right)$. We claim that $\gamma=\gamma^{\prime}$.

Suppose that $\gamma \neq \gamma^{\prime}$. Without loss of generality, we can assume that $\gamma$ is above $\gamma^{\prime}$. Suppose that $\gamma^{\prime}$ is occupied by $j>1$ in $\mathfrak{s}$. Let $[\widetilde{\mu}]:=[\mu] \cup$ Shape $\left(\mathfrak{s} \downarrow_{j}\right)$. By definition, $\widetilde{\mu}$ is an $l$-special partition and $\gamma^{\prime}$ is a removable node of $\widetilde{\mu}$. We have that

$$
1 \leq a<a^{\prime} \leq \ell(\widetilde{\mu}), \quad \widetilde{\mu}_{\ell(\widetilde{\mu})} \leq b^{\prime}<b \leq \widetilde{\mu}_{1},
$$

and (as $\widetilde{\mu}$ is $l$-special) $\widetilde{\mu}_{1}-\widetilde{\mu}_{\ell(\widetilde{\mu})} \leq l-\ell(\widetilde{\mu})$. Hence

$$
\begin{aligned}
1<\operatorname{Res}(\gamma)-\operatorname{Res}\left(\gamma^{\prime}\right) & =(b-a)-\left(b^{\prime}-a^{\prime}\right) \leq \widetilde{\mu}_{1}-\widetilde{\mu}_{\ell(\widetilde{\mu})}+\ell(\widetilde{\mu})-1 \\
& \leq l-1,
\end{aligned}
$$

which is a contradiction.

Now use induction on $n$, our claim follows immediately. By remark 2.11, it is easy to see that the same is true if one replaces " $l$-special" by " $(l, m)$-special".

Let $\nu=\lambda / \mu$ be an $(l, m)$-special skew shape (with respect to $\mu$ ) with $n$ nodes. The symmetric group $\mathfrak{S}_{n}$ acts from right on the set of $\nu$-tableaux. Given an $(l, m)$-special standard $\lambda / \mu$-tableau $\mathfrak{t}$, we remark that even if $\mathfrak{t} s_{i}$ (where $1 \leq i<$ $n)$ is a standard tableau, $\mathfrak{t}_{i}$ does not necessarily have to be an $(l, m)$-special standard $\lambda / \mu$-tableau. For example, let $\mathfrak{t}$ be the first $(8,5)$-special standard $\left(4,3^{2}, 1^{2}\right) /\left(3,2,1^{2}\right)$-tableau in Example 2.10, 


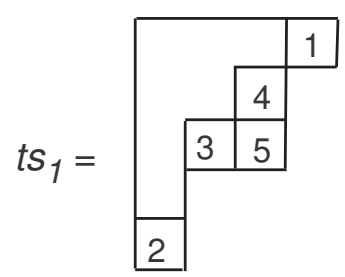

is not a $(8,5)$-special standard $\left(4,3^{2}, 1^{2}\right) /\left(3,2,1^{2}\right)$-tableau. But we have

Lemma 2.17 Let $\nu=\lambda / \mu$ be an $(l, m)$-special skew shape with $n$ nodes. Let $\mathfrak{t}$ be an $(l, m)$-special standard $\lambda / \mu$-tableau. Let $k$ be an integer with $1 \leq k<n$, and $[\widetilde{\lambda}]=[\mu] \cup \operatorname{Shape}\left(\mathfrak{t} \downarrow_{k+1}\right)$. Let $\gamma\left(\right.$ resp. $\left.\gamma^{\prime}\right)$ be the node in $[\nu]$ occupied by $k$ (resp. by $k+1$ ) in $\mathfrak{t}$. Suppose that $\mathfrak{t} s_{k}$ is a standard but not an $(l, m)$-special standard $\lambda / \mu$-tableau. Then $m=\ell(\widetilde{\lambda})=\ell(\lambda)$, and

$$
\gamma=\left(m, \widetilde{\lambda}_{m}\right), \quad \gamma^{\prime}=\left(1, \widetilde{\lambda}_{1}\right), \quad \operatorname{Res}_{\mathfrak{t}}(k)-\operatorname{Res}_{\mathfrak{t}}(k+1)=-l+1 .
$$

Proof. Since $\mathfrak{t}_{k}$ is a standard tableau, it follows that $\gamma$ and $\gamma^{\prime}$ can not be in the same row or the same column of $[\lambda]$. In particular, both $\gamma$ and $\gamma^{\prime}$ are removable nodes of $[\widetilde{\lambda}]$. Write $\gamma=(a, b), \gamma^{\prime}=\left(a^{\prime}, b^{\prime}\right)$. By definition, $\widetilde{\lambda}$ is an $(l, m)$-special partition. Since $\mathfrak{t} s_{k}$ is not $(l, m)$-special standard with respect to $\mu$, it follows that $m=\ell(\widetilde{\lambda})=\ell(\lambda)$, and either $\gamma=\left(m, \widetilde{\lambda}_{m}\right), \gamma^{\prime}=\left(1, \widetilde{\lambda}_{1}\right)$ or $\gamma=\left(1, \widetilde{\lambda}_{1}\right), \gamma^{\prime}=\left(m, \widetilde{\lambda}_{m}\right)$.

Suppose that $\gamma=\left(1, \widetilde{\lambda}_{1}\right), \gamma^{\prime}=\left(m, \widetilde{\lambda}_{m}\right)$. Since $[\mu] \cup \operatorname{Shape}\left(\mathfrak{t} \downarrow_{k}\right)$ is an $(l, m)$ special partition, we have $\widetilde{\lambda}_{1}-\left(\widetilde{\lambda}_{m}-1\right) \leq l-m$. In particular, $\left(\widetilde{\lambda}_{1}-1\right)-\widetilde{\lambda}_{m} \leq$ $l-m-2<l-m$, which contradicts to the fact that $\mathrm{t}_{k}$ is not $(l, m)$-special with respect to $\mu$. Therefore, $\gamma=\left(m, \widetilde{\lambda}_{m}\right)$, and $\gamma^{\prime}=\left(1, \widetilde{\lambda}_{1}\right)$.

Since $\mathfrak{t}$ is $(l, m)$-special standard with respect to $\mu$, we have $\widetilde{\lambda}_{1}-\widetilde{\lambda}_{m} \leq l-m$. On the other hand, as $t_{k}$ is not $(l, m)$-special standard with respect to $\mu$, we have $\widetilde{\lambda}_{1}-\left(\widetilde{\lambda}_{m}-1\right)>l-m$. It follows that $\widetilde{\lambda}_{1}-\widetilde{\lambda}_{m}=l-m$. In particular, $\operatorname{Res}_{\mathfrak{t}}(k)-\operatorname{Res}_{\mathfrak{t}}(k+1)=\left(\widetilde{\lambda}_{m}-m\right)-\left(\widetilde{\lambda}_{1}-1\right)=-l+1$, as required.

Lemma 2.18 Let $\nu=\lambda / \mu$ be an $(l, m)$-special skew shape with $n$ nodes. Let $\mathfrak{t}$ be an $(l, m)$-special standard $\lambda / \mu$-tableau. Let $k$ be an integer with $1 \leq k<n$. Let $\gamma\left(\right.$ resp. $\left.\gamma^{\prime}\right)$ be the node in $[\nu]$ occupied by $k$ (resp. by $k+1$ ) in $\mathfrak{t}$. Suppose that $\mathfrak{t} s_{k}$ is an $(l, m)$-special standard $\lambda / \mu$-tableau. Then

$$
\left|\operatorname{Res}_{\mathfrak{t}}(k)-\operatorname{Res}_{\mathfrak{t}}(k+1)\right| \notin\{0,1, l-1\} .
$$

Proof. Write $\gamma=(a, b), \gamma^{\prime}=\left(a^{\prime}, b^{\prime}\right)$. By Lemma 2.15, it suffices to show that $\left|\operatorname{Res}_{\mathfrak{t}}(k)-\operatorname{Res}_{\mathfrak{t}}(k+1)\right| \neq l-1$. 
Let $[\widetilde{\lambda}]=[\mu] \cup$ Shape $\left(\mathfrak{t} \downarrow_{k+1}\right)$. As $\mathfrak{t}_{k}$ is standard, we know that both $\gamma, \gamma^{\prime}$ are removable nodes of $[\widetilde{\lambda}]$. Since $\mathfrak{t}_{k}$ is an $(l, m)$-special standard $\lambda / \mu$-tableau, it follows that $1<\ell(\widetilde{\lambda}) \leq m$, and $\widetilde{\lambda}_{1}-\widetilde{\lambda}_{m} \leq l-m$. Hence

$$
0<\left|\operatorname{Res}(\gamma)-\operatorname{Res}\left(\gamma^{\prime}\right)\right| \leq \widetilde{\lambda}_{1}-\widetilde{\lambda}_{m}+m-1 \leq l-1,
$$

with equality holds only when $\ell(\widetilde{\lambda})=m,\left\{\gamma, \gamma^{\prime}\right\}=\left\{\left(1, \widetilde{\lambda}_{1}\right),\left(m, \widetilde{\lambda}_{m}\right)\right\}, \widetilde{\lambda}_{1}-\widetilde{\lambda}_{m}=$ $l-m$.

Case 1. $\gamma^{\prime}=\left(1, \widetilde{\lambda}_{1}\right), \gamma=\left(m, \widetilde{\lambda}_{m}\right)$.

If $\widetilde{\lambda}_{m}>1$, then as $[\mu] \cup \operatorname{Shape}\left(\mathfrak{t}_{k} \downarrow_{k}\right)$ is an $(l, m)$-special partition, it follows that $\widetilde{\lambda}_{1}-\left(\widetilde{\lambda}_{m}-1\right) \leq l-m$, which contradicts to fact that $\widetilde{\lambda}_{1}-\widetilde{\lambda}_{m}=l-m$. Hence $\widetilde{\lambda}_{m}=1$. As $[\mu] \cup \operatorname{Shape}\left(\mathfrak{t}_{k} \downarrow_{k}\right)$ is an $(l, m)$-special partition, we have that $\widetilde{\lambda}_{1}=\widetilde{\lambda}_{1}-0 \leq l-m$, which again contradicts to fact that $\widetilde{\lambda}_{1}-\widetilde{\lambda}_{m}=l-m$.

Case 2. $\gamma=\left(1, \widetilde{\lambda}_{1}\right), \gamma^{\prime}=\left(m, \widetilde{\lambda}_{m}\right)$.

If $\widetilde{\lambda}_{m}>1$, then as $[\mu] \cup \operatorname{Shape}\left(\mathfrak{t} \downarrow_{k}\right)$ is an $(l, m)$-special partition, it follows that $\widetilde{\lambda}_{1}-\left(\widetilde{\lambda}_{m}-1\right) \leq l-m$, which contradicts to fact that $\widetilde{\lambda}_{1}-\widetilde{\lambda}_{m}=l-m$. Hence $\widetilde{\lambda}_{m}=1$. As $[\mu] \cup$ Shape $\left(\mathfrak{t} \downarrow_{k}\right)$ is an $(l, m)$-special partition, we have that $\widetilde{\lambda}_{1}=\widetilde{\lambda}_{1}-0 \leq l-m$, which again contradicts to fact that $\widetilde{\lambda}_{1}-\widetilde{\lambda}_{m}=l-m$. This completes the proof of the lemma.

Lemma 2.19 Let $\nu=\lambda / \mu$ be an $l$-special skew shape with $n$ nodes. Let $\mathfrak{t}$ be an $l$-special standard $\lambda / \mu$-tableau. Let $k$ be an integer with $1 \leq k<n$, and $[\widetilde{\lambda}]=[\mu] \cup \operatorname{Shape}\left(\mathfrak{t} \downarrow_{k+1}\right)$. Let $\gamma\left(\right.$ resp. $\left.\gamma^{\prime}\right)$ be the node in $[\nu]$ occupied by $k$ (resp. by $k+1$ ) in $\mathfrak{t}$. Suppose that $\mathfrak{t}_{k}$ is a standard but not an $l$-special standard $\lambda / \mu$-tableau. Then

$$
\gamma=\left(\ell(\widetilde{\lambda}), \widetilde{\lambda}_{\ell(\widetilde{\lambda})}\right), \quad \gamma^{\prime}=\left(1, \widetilde{\lambda}_{1}\right), \quad \widetilde{\lambda}_{\ell(\widetilde{\lambda})}>1,
$$

and $\operatorname{Res}_{\mathfrak{t}}(k)-\operatorname{Res}_{\mathfrak{t}}(k+1)=-l+1$.

Proof. Since $\mathfrak{t} s_{k}$ is a standard tableau, it follows that $\gamma$ and $\gamma^{\prime}$ can not be in the same row or the same column of $[\lambda]$. In particular, both $\gamma$ and $\gamma^{\prime}$ are removable nodes of $[\widetilde{\lambda}]$. Write $\gamma=(a, b), \gamma^{\prime}=\left(a^{\prime}, b^{\prime}\right)$. By definition, $\widetilde{\lambda}$ is an $l$-special partition. Since $t_{k}$ is not $l$-special standard with respect to $\mu$, it follows that either $\gamma=\left(\ell(\widetilde{\lambda}), \widetilde{\lambda}_{\ell(\widetilde{\lambda})}\right), \gamma^{\prime}=\left(1, \widetilde{\lambda}_{1}\right)$ or $\gamma=\left(1, \widetilde{\lambda}_{1}\right), \gamma^{\prime}=\left(\ell(\widetilde{\lambda}), \widetilde{\lambda}_{\ell(\widetilde{\lambda})}\right)$.

Suppose that $\gamma=\left(1, \widetilde{\lambda}_{1}\right), \gamma^{\prime}=\left(\ell(\widetilde{\lambda}), \widetilde{\lambda}_{\ell(\widetilde{\lambda})}\right)$. If $\widetilde{\lambda}_{\ell(\widetilde{\lambda})}>1$, then as $[\mu] \cup \operatorname{Shape}\left(\mathfrak{t} \downarrow_{k}\right.$ ) is an $l$-special partition, we have $\widetilde{\lambda}_{1}-\left(\widetilde{\lambda}_{\ell(\widetilde{\lambda})}-1\right) \leq l-\ell(\widetilde{\lambda})$. In particular, $\left(\widetilde{\lambda}_{1}-1\right)-\lambda_{\ell(\widetilde{\lambda})} \leq l-\ell(\widetilde{\lambda})-2<l-\ell(\widetilde{\lambda})$, which contradicts to the fact that $\mathfrak{t}_{k}$ is not $l$-special with respect to $\mu$; If $\tilde{\lambda}_{\ell(\tilde{\lambda})}=1$, then as $\tilde{\lambda}$ is $l$-special, we have 
that $\widetilde{\lambda}_{1}-1 \leq l-\ell(\widetilde{\lambda})$. In this case, $\widetilde{\lambda}_{1}-1-\widetilde{\lambda}_{\ell(\widetilde{\lambda})}=\widetilde{\lambda}_{1}-2<l-\ell(\widetilde{\lambda})$, which still contradicts to the fact that $\mathrm{t}_{k}$ is not $l$-special with respect to $\mu$; Therefore, $\gamma=\left(\ell(\widetilde{\lambda}), \widetilde{\lambda}_{\ell(\tilde{\lambda})}\right), \gamma^{\prime}=\left(1, \widetilde{\lambda}_{1}\right)$.

Since $\tilde{\lambda}$ is $l$-special, we have $\tilde{\lambda}_{1}-\widetilde{\lambda}_{\ell(\tilde{\lambda})} \leq l-\ell(\widetilde{\lambda})$. If $\widetilde{\lambda}_{\ell(\widetilde{\lambda})}=1$, then $\widetilde{\lambda}_{1}-$ $1 \leq l-\ell(\widetilde{\lambda})$. But as $\mathfrak{t}_{k}$ is not $l$-special with respect to $\mu$, we also have that $\widetilde{\lambda}_{1}-\widetilde{\lambda}_{\ell(\tilde{\lambda})-1}>l-(\ell(\widetilde{\lambda})-1)$. It follows that $\widetilde{\lambda}_{1}-1 \geq \widetilde{\lambda}_{1}-\widetilde{\lambda}_{\ell(\widetilde{\lambda})-1}>l-\ell(\widetilde{\lambda})+1$, which is impossible. Hence $\widetilde{\lambda}_{\ell(\tilde{\lambda})}>1$. As $t_{k}$ is not $l$-special with respect to $\mu$, we have $\widetilde{\lambda}_{1}-\left(\widetilde{\lambda}_{\ell(\widetilde{\lambda})}-1\right)>l-\ell(\widetilde{\lambda})$. It follows that $\tilde{\lambda}_{1}-\widetilde{\lambda}_{\ell(\widetilde{\lambda})}=l-\ell(\widetilde{\lambda})$. In particular,

$$
\operatorname{Res}_{\mathfrak{t}}(k)-\operatorname{Res}_{\mathfrak{t}}(k+1)=\left(\widetilde{\lambda}_{\ell(\widetilde{\lambda})}-\ell(\widetilde{\lambda})\right)-\left(\widetilde{\lambda}_{1}-1\right)=-l+1,
$$

as required.

For any two standard $\lambda$-tableaux $\mathfrak{s}, \mathfrak{t}$, it is well-known that there exist integers $1 \leq i_{1}, \cdots, i_{k}<n$, such that $\mathfrak{s} s_{i_{1}} s_{i_{2}} \cdots s_{i_{k}}=\mathfrak{t}$ and for each $1 \leq j \leq k$, $\mathfrak{s} s_{i_{1}} s_{i_{2}} \cdots s_{i_{j}}$ is a standard tableau. The following two theorems tell us that the same is true if one replace "partitions" by " $l$-special or $(l, m)$-special skew shapes", and "standard tableaux" by " $l$-special or $(l, m)$-special standard tableaux" respectively.

Theorem 2.20 Let $\nu=\lambda / \mu$ be an $l$-special skew shape with $n$ nodes. Let $\mathfrak{s , t}$ be two $l$-special standard $\lambda / \mu$-tableaux. Then there exist integers $1 \leq i_{1}, \cdots, i_{k}<$ $n$, such that for $j=1, \cdots, k, \mathfrak{t}_{0}=\mathfrak{s}, \mathfrak{t}_{j}=\mathfrak{t}_{j-1} s_{i_{j}}, \mathfrak{t}_{k}=\mathfrak{t}$, and each $\mathfrak{t}_{j}$ is an $l$-special standard $\lambda / \mu$-tableau.

Proof. We use induction on $n$. If $n=1$, there is nothing to prove. Suppose that the conclusion is true for $n-1$. We want to prove that it is also true for $n$. Let $\nu=\lambda / \mu$ be an $l$-special skew shape (with respect to $\mu$ ) with $n$ nodes. Let $\mathfrak{s}, \mathfrak{t}$ be two $l$-special standard $\lambda / \mu$-tableaux. Let $\gamma$ be the node which is occupied by $n$ in $\mathfrak{s}$, and let $\gamma^{\prime}$ be the node which is occupied by $n$ in $\mathfrak{t}$. If $\gamma=\gamma^{\prime}$, then the theorem follows immediately from induction hypothesis. Now suppose that $\gamma \neq \gamma^{\prime}$.

Without loss of generality, we can assume that $\gamma^{\prime}$ is above $\gamma$. In particular, $\ell(\lambda)>1$. Suppose that $\gamma^{\prime}$ is the node occupied by $k$ in $\mathfrak{s}$. Then $1 \leq k \leq n-1$. Let $\gamma_{1}$ be the node occupied by $k+1$ in $\mathfrak{s}$. As $\gamma^{\prime}$ is a removable node of $[\lambda]$ and $\mathfrak{s}$ is a standard tableau, $\gamma_{1}$ is not in the same row or the same column as $\gamma^{\prime}$. In particular, $\mathfrak{t}_{1}:=\mathfrak{s} s_{k}$ is a standard tableau. We claim that $\mathfrak{t}_{1}$ must be $l$-special with respect to $\mu$.

Suppose that $\mathfrak{t}_{1}$ is not $l$-special with respect to $\mu$. By Lemma 2.19, we know that $\gamma_{1}$ is above $\gamma^{\prime}$ and $\operatorname{Res}\left(\gamma^{\prime}\right)-\operatorname{Res}\left(\gamma_{1}\right)=-l+1$. 
We write $\gamma_{1}=(a, b), \gamma^{\prime}=\left(a^{\prime}, b^{\prime}\right)$, and $[\widetilde{\lambda}]=[\mu] \cup \operatorname{Shape}\left(\mathfrak{t} \downarrow_{n-1}\right)$. Then $\tilde{\lambda}$ is $l$-special and we have that $\widetilde{\lambda}_{1}-\widetilde{\lambda}_{\ell(\widetilde{\lambda})} \leq l-\ell(\widetilde{\lambda})$. Note that $\gamma^{\prime}$ is occupied by $n$ in t. It follows that

$$
1 \leq a, a^{\prime} \leq \ell(\widetilde{\lambda}), \quad \widetilde{\lambda}_{\ell(\widetilde{\lambda})}<b, b^{\prime} \leq \widetilde{\lambda}_{1}
$$

Hence

$$
0<\operatorname{Res}\left(\gamma_{1}\right)-\operatorname{Res}\left(\gamma^{\prime}\right)<\widetilde{\lambda}_{1}-\widetilde{\lambda}_{\ell(\widetilde{\lambda})}-1+\ell(\widetilde{\lambda}) \leq l-1,
$$

which is a contradiction. This proves our claim.

Now $\gamma^{\prime}$ is occupied by $k+1$ in $\mathfrak{t}_{1}$. Repeating the use of Lemma 2.19 , it is easy to see that there exist integers $1 \leq i_{1}, \cdots, i_{k}<n$, such that for $j=1, \cdots, k$, $\mathfrak{t}_{0}=\mathfrak{s}, \mathfrak{t}_{j}=\mathfrak{t}_{j-1} s_{i_{j}}, \mathfrak{t}_{k}=\mathfrak{u}$, and each $\mathfrak{t}_{j}$ is an $l$-special standard $\lambda / \mu$-tableau and $\gamma^{\prime}$ is occupied by $n$ in $\mathfrak{u}$.

Now note that in either of the two $l$-special standard $\lambda / \mu$-tableaux $\mathfrak{u}, \mathfrak{t}, \gamma^{\prime}$ is occupied by $n$. Hence we can use induction hypothesis, and the theorem follows immediately.

Theorem 2.21 Let $\nu=\lambda / \mu$ be an $(l, m)$-special skew shape with $n$ nodes. Let $\mathfrak{s}, \mathfrak{t}$ be two $(l, m)$-special standard $\lambda / \mu$-tableaux. Then there exist integers $1 \leq i_{1}, \cdots, i_{k}<n$, such that for $j=1, \cdots, k, \mathfrak{t}_{0}=\mathfrak{s}, \mathfrak{t}_{j}=\mathfrak{t}_{j-1} s_{i_{j}}, \mathfrak{t}_{k}=\mathfrak{t}$ are all standard $\nu$-tableaux, and

$$
\left|\operatorname{Res}_{t_{j-1}}\left(i_{j}\right)-\operatorname{Res}_{\mathfrak{t}_{j-1}}\left(i_{j}+1\right)\right| \notin\{0,1, l-1\} .
$$

In particular (by Lemma 2.17), for each $0 \leq j \leq k$, each $\mathfrak{t}_{j}$ is an $(l, m)$-special standard $\lambda / \mu$-tableau.

Proof. We use induction on $n$. If $n=1$, there is nothing to prove. Suppose that the conclusion is true for $n-1$. We want to prove that it is also true for $n$. Let $\nu=\lambda / \mu$ be an $(l, m)$-special skew shape (with respect to $\mu$ ) with $n$ nodes. Let $\mathfrak{s}, \mathfrak{t}$ be two $(l, m)$-special standard $\lambda / \mu$-tableaux. Let $\gamma$ be the node which is occupied by $n$ in $\mathfrak{s}$, and let $\gamma^{\prime}$ be the node which is occupied by $n$ in $\mathfrak{t}$. If $\gamma=\gamma^{\prime}$, then the theorem follows immediately from induction hypothesis. Now suppose that $\gamma \neq \gamma^{\prime}$.

Without loss of generality, we can assume that $\gamma^{\prime}$ is above $\gamma$. In particular, $\ell(\lambda)>1$. Write $\gamma=(a, b), \gamma^{\prime}=\left(a^{\prime}, b^{\prime}\right)$. Then

$$
1 \leq a^{\prime}<a \leq \ell(\lambda), \quad \lambda_{\ell(\lambda)} \leq b<b^{\prime} \leq \lambda_{1} .
$$

Suppose that $\gamma^{\prime}$ is the node occupied by $k$ in $\mathfrak{s}$. Then $1 \leq k \leq n-1$. Let $\gamma_{1}$ be the node occupied by $k+1$ in $\mathfrak{s}$. As $\gamma^{\prime}$ is a removable node of $[\lambda]$ and $\mathfrak{s}$ is a standard tableau, $\gamma_{1}$ is not in the same row or the same column as $\gamma^{\prime}$. In particular, $\mathfrak{t}_{1}:=\mathfrak{s} s_{k}$ is a standard tableau. Since $\lambda$ is $(l, m)$-special (and hence $l$-special), we have $\ell(\lambda) \leq m$ and $\lambda_{1}-\lambda_{\ell(\lambda)} \leq l-\ell(\lambda)$. In particular, $a^{\prime}<m$. Applying Lemma 
2.17 and Lemma 2.18 , it is easy to see that $\mathfrak{t}_{1}$ is also $(l, m)$-special standard with respect to $\mu$, and

$$
\left|\operatorname{Res}_{\mathfrak{s}}(k)-\operatorname{Res}_{\mathfrak{s}}(k+1)\right| \notin\{0,1, l-1\} .
$$

Now note that $\gamma^{\prime}=\left(a^{\prime}, b^{\prime}\right)$ (with $a^{\prime}<m$ ) is occupied by $k+1$ in $\mathfrak{t}_{1}$. Repeating the use of Lemma 2.17 and Lemma 2.18, it is easy to see that there exists integers $1 \leq i_{1}, \cdots, i_{n-k}<n$, such that for $j=1, \cdots, n-k, \mathfrak{t}_{0}=\mathfrak{s}, \mathfrak{t}_{j}=\mathfrak{t}_{j-1} s_{i_{j}}, \mathfrak{t}_{n-k}=\mathfrak{u}$ are all $(l, m)$-special standard (with respect to $\mu$ ), and

$$
\left|\operatorname{Res}_{\mathfrak{t}_{j-1}}\left(i_{j}\right)-\operatorname{Res}_{\mathfrak{t}_{j-1}}\left(i_{j}+1\right)\right| \notin\{0,1, l-1\} .
$$

Now note that in either of the two $(l, m)$-special standard $\lambda / \mu$-tableaux $\mathfrak{u}, \mathfrak{t}$, $\gamma^{\prime}$ is occupied by $n$. Hence we can use induction hypothesis, and the theorem follows immediately.

\section{The indecomposable module $I(\lambda, \mu)$}

Let $K, q, l$ be defined as before. In this section, we shall construct, for each $l$-special skew shape $\lambda / \mu$, an indecomposable module $I(\lambda, \mu)$ over the affine Hecke algebra $\mathcal{H}_{n}^{\text {aff }}$ explicitly. Our constructions are based on the Young seminormal representations for the affine Hecke algebras (see [Ho], [AK], [HaR], [R1-4]).

Let $\nu=\lambda / \mu$ be an $l$-special skew shape with respect to $\mu$. We use our definition of Young diagram to fix a coordinate plane. ${ }^{5}$ We define $I(\lambda, \mu)$ to be a $K$-linear space with a basis labelled by all the $l$-special standard $\lambda / \mu$-tableaux, that is

$$
I(\lambda, \mu):=K \text {-span }\left\{v_{\mathfrak{t}} \mid \mathfrak{t} \text { is an } l \text {-special standard } \lambda / \mu \text {-tableau }\right\} .
$$

Recall the definition of the affine Hecke algebra $\mathcal{H}_{n}^{\text {aff }}$ in 2.1. For each integer $i, j$ with $1 \leq i<n, 1 \leq j \leq n$, and for each $l$-special standard $\lambda / \mu$-tableau $\mathfrak{t}$, we define

$$
\begin{aligned}
v_{\mathfrak{t}} T_{i} & =\frac{q-1}{1-q^{\operatorname{res}_{\mathfrak{t}}(i)-\operatorname{res}_{\mathfrak{t}}(i+1)}} v_{\mathfrak{t}}+\left(1+\frac{q-1}{1-q^{\operatorname{res}_{\mathfrak{t}}(i)-\operatorname{res}_{\mathfrak{t}}(i+1)}}\right) v_{\mathfrak{t s}_{i}}, \\
v_{\mathfrak{t}} X_{j} & =q^{\operatorname{res}_{\mathfrak{t}}(j)} v_{\mathfrak{t}}
\end{aligned}
$$

if $q \neq 1$; or

$$
\begin{aligned}
v_{\mathfrak{t}} s_{i} & =\frac{1}{\operatorname{res}_{\mathfrak{t}}(i+1)-\operatorname{res}_{\mathfrak{t}}(i)} v_{\mathfrak{t}}+\left(1+\frac{1}{\operatorname{res}_{\mathfrak{t}}(i+1)-\operatorname{res}_{\mathfrak{t}}(i)}\right) v_{\mathfrak{t s}_{i}}, \\
v_{\mathfrak{t}} X_{j} & =\operatorname{res}_{\mathfrak{t}}(j) v_{\mathfrak{t}}
\end{aligned}
$$

\footnotetext{
${ }^{5}$ If we use a new coordinate plane obtained by parallel translation and define Res, res functions with respect to that new coordinate plane, then the same procedure in this section will give rise to a new family indecomposable modules. They are in fact the twist of the old ones by certain automorphism of $\mathcal{H}_{n}^{\text {aff }}$. The same remark applies to our construction in Section 4.
} 
if $q=1$,where if $t_{i}$ is not an $l$-special standard $\lambda / \mu$-tableau, then we define $v_{\mathfrak{t s}_{i}}=0$. By Lemma 2.15, both (3.2) and (3.3) are well-defined.

Theorem 3.4 The above action extends to a well-defined action of $\mathcal{H}_{n}^{\text {aff }}$ on the $I(\lambda, \mu)$.

Proof. This can be verified by case by case arguments as in [R2].

Theorem 3.6 For each $l$-special skew shape $\nu=\lambda / \mu$ (with respect to $\mu$ ), $I(\lambda, \mu)$ is an indecomposable module over the affine Hecke algebra $\mathcal{H}_{n}^{\text {aff }}$.

Proof. It is easy to see that for each 2 -special skew shapes $\nu$, there is only one 2 -special standard $\nu$-tableau. In that case the conclusion is obvious. So we can assume that $l>2$. Let $\nu=\lambda / \mu$ be an $l$-special skew shape. Recall that $\operatorname{Std}_{l}(\lambda, \mu)=\{\mathfrak{t} \mid \mathfrak{t}$ is an $l$-special standard $\lambda / \mu$-tableau $\}$. For any $l$-special standard $\lambda / \mu$-tableau $\mathfrak{t}$, we define

$$
\Theta_{\mathfrak{t}}:=\prod_{i=1}^{n} \prod_{\substack { u \\
\begin{subarray}{c}{u \\
\operatorname{res}_{\mathfrak{u}}(i) \neq \operatorname{St}_{l}(\lambda, \mu){ u \\
\begin{subarray} { c } { u \\
\operatorname { r e s } _ { \mathfrak { u } } ( i ) \neq \operatorname { S t } _ { l } ( \lambda , \mu ) } }\end{subarray}} \frac{X_{i}-q^{\operatorname{res}_{\mathfrak{u}}(i)}}{q^{\operatorname{res}_{\mathfrak{t}}(i)}-q^{\operatorname{res}_{\mathfrak{u}}(i)}},
$$

if $q \neq 1$; or

$$
\Theta_{\mathfrak{t}}:=\prod_{i=1}^{n} \prod_{\substack{\mathfrak{u} \in \operatorname{Sdd}_{l}(\lambda, \mu) \\ \operatorname{res}_{\mathfrak{u}}(i) \neq \operatorname{res}_{\mathfrak{t}}(i)}} \frac{X_{i}-\operatorname{res}_{\mathfrak{u}}(i)}{\operatorname{res}_{\mathfrak{t}}(i)-\operatorname{res}_{\mathfrak{u}}(i)}
$$

if $q=1$.

By Lemma 2.16, for any $l$-special standard $\lambda / \mu$-tableaux $\mathfrak{u} \neq \mathfrak{v}$, there exists an integer $k$ with $1 \leq k \leq n$, such that $\operatorname{res}_{\mathfrak{u}}(k) \neq \operatorname{res}_{\mathfrak{v}}(k)$. It follows that $v_{\mathfrak{v}} \Theta_{\mathfrak{u}}=\delta_{\mathfrak{u}, \mathfrak{v}} v_{\mathfrak{v}}$.

For any $0 \neq v \in I(\lambda, \mu)$, we write $v=\sum_{\mathfrak{t} \in \operatorname{Std}_{l}(\lambda, \mu)} a_{\mathfrak{t}} v_{\mathfrak{t}}$. Applying the operator $\Theta_{\mathfrak{t}}$, it is easy to see that $a_{\mathfrak{t}} \neq 0$ implies that $v_{\mathfrak{t}} \in v \mathcal{H}_{n}^{\text {aff }}$. In particular, this proves that every submodule of $I(\lambda, \mu)$ is spanned by the base elements it contains.

Now let $\mathfrak{t}$ be an $l$-special standard $\lambda / \mu$-tableau, and let $k$ be an integer with $1 \leq k<n$ such that $\mathfrak{t}_{k}$ is also an $l$-special standard $\lambda / \mu$-tableau. We have

Case 1 .

$\left|\operatorname{Res}_{\mathfrak{t}}(k)-\operatorname{Res}_{\mathfrak{t}}(k+1)\right| \notin\{1, l-1\}$.

Since

$$
\begin{aligned}
v_{\mathfrak{t}} T_{k} & =\frac{q-1}{1-q^{\text {res }_{\mathfrak{t}}(k)-\text { res }_{\mathfrak{t}}(k+1)}} v_{\mathfrak{t}}+\left(\frac{q-q^{\text {res }_{\mathfrak{t}}(k)-\text { res }_{\mathfrak{t}}(k+1)}}{1-q^{\text {res }_{\mathfrak{t}}(k)-\operatorname{res}_{\mathfrak{t}}(k+1)}}\right) v_{\mathfrak{t s}_{k}}, \\
v_{\mathfrak{t s}_{k}} T_{k} & =\frac{q-1}{1-q^{\text {res }_{\mathfrak{t}}(k+1)-\operatorname{res}_{\mathfrak{t}}(k)}} v_{\mathfrak{t}_{k}}+\left(\frac{q-q^{\text {res }_{\mathfrak{t}}(k+1)-\operatorname{res}_{\mathfrak{t}}(k)}}{1-q^{\text {res }_{\mathfrak{t}}(k+1)-\operatorname{res}_{\mathfrak{t}}(k)}}\right) v_{\mathfrak{t}},
\end{aligned}
$$


if $q \neq 1$; or

$$
\begin{aligned}
v_{\mathfrak{t}} s_{k} & =\frac{1}{\operatorname{res}_{\mathfrak{t}}(k+1)-\operatorname{res}_{\mathfrak{t}}(k)} v_{\mathfrak{t}}+\left(\frac{1+\operatorname{res}_{\mathfrak{t}}(k+1)-\operatorname{res}_{\mathfrak{t}}(k)}{\operatorname{res}_{\mathfrak{t}}(k+1)-\operatorname{res}_{\mathfrak{t}}(k)}\right) v_{\mathfrak{t}_{k}}, \\
v_{\mathfrak{t}_{k}} s_{k} & =\frac{1}{\operatorname{res}_{\mathfrak{t}}(k)-\operatorname{res}_{\mathfrak{t}}(k+1)} v_{\mathfrak{t}_{k}}+\left(\frac{1+\operatorname{res}_{\mathfrak{t}}(k)-\operatorname{res}_{\mathfrak{t}}(k+1)}{\operatorname{res}_{\mathfrak{t}}(k)-\operatorname{res}_{\mathfrak{t}}(k+1)}\right) v_{\mathfrak{t}},
\end{aligned}
$$

if $q=1$, It follows that $v_{\mathfrak{t s}_{k}} \in v_{\mathfrak{t}} \mathcal{H}_{n}^{\text {aff }}$ and $v_{\mathfrak{t}} \in v_{\mathfrak{t s}_{k}} \mathcal{H}_{n}^{\text {aff }}$.

Case 2. $\operatorname{res}_{\mathfrak{t}}(k)-\operatorname{res}_{\mathfrak{t}}(k+1)=1$.

It follows (as above) that $v_{\mathfrak{t}} \in v_{\mathfrak{t s}_{k}} \mathcal{H}_{n}^{\text {aff }}$.

Case 3. $\operatorname{res}_{\mathfrak{t}}(k)-\operatorname{res}_{\mathfrak{t}}(k+1)=l-1$.

It follows (as above) that $v_{\mathfrak{t s}_{k}} \in v_{\mathfrak{t}} \mathcal{H}_{n}^{\text {aff }}$.

Now applying Theorem 2.20, and noting that each submodule of $I(\lambda, \mu)$ is spanned by the base elements it contains, the theorem follows immediately.

Note that the proof of the above theorem also yields a combinatorial way to determine the composition series for each indecomposable module $I(\lambda, \mu)$.

Example 3.9 Suppose $n=3, l=4, \nu=(3,1) /(1)$. The following are all the 4 -special standard $\lambda / \mu$-tableaux.
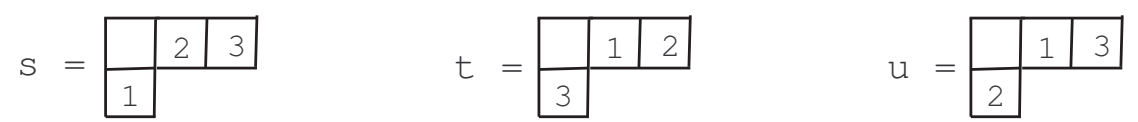

The subspace spanned by $v_{\mathfrak{s}}, v_{\mathfrak{u}}$ is a 2-dimensional irreducible module over $\mathcal{H}_{n}^{\text {aff }}$, and the quotient space $I(\lambda, \mu) /\left(K v_{\mathfrak{s}} \oplus K v_{\mathfrak{u}}\right)$ is an 1-dimesional irreducible module.

\section{The irreducible module $D(\lambda, \mu)$}

Let $K, q, l, m$ be defined as before. In this section, we shall construct, for each $(l, m)$-special skew shape $\nu=\lambda / \mu$, an irreducible module $D(\lambda, \mu)$ over the affine Hecke algebra $\mathcal{H}_{n}^{\text {aff }}$ explicitly. Our constructions are also based on the Young seminormal representations for the affine Hecke algebras (see [Ho], [AK], [HaR], $[\mathrm{R} 1-4])$.

Let $\nu=\lambda / \mu$ be an $(l, m)$-special skew shape with respect to $\mu$. We use our definition of Young diagram to fix a coordinate plane. We define $D(\lambda, \mu)$ to be 
a $K$-linear space with a basis labelled by all the $(l, m)$-special standard $\lambda / \mu$ tableaux, that is

$$
D(\lambda, \mu):=K \text {-span }\left\{v_{\mathfrak{t}} \mid \mathfrak{t} \text { is an }(l, m) \text {-special standard } \lambda / \mu \text {-tableau }\right\} .
$$

Recall the definition of the affine Hecke algebra $\mathcal{H}_{n}^{\text {aff }}$ in 2.1. For each integer $i, j$ with $1 \leq i<n, 1 \leq j \leq n$, and for each $(l, m)$-special standard $\nu$-tableau $\mathfrak{t}$, we define

$$
\begin{aligned}
v_{\mathfrak{t}} T_{i} & =\frac{q-1}{1-q^{\text {rest }_{\mathfrak{t}}(i)-\operatorname{res}_{\mathfrak{t}}(i+1)}} v_{\mathfrak{t}}+\left(1+\frac{q-1}{1-q^{\operatorname{res}_{\mathfrak{t}}(i)-\operatorname{res}_{\mathfrak{t}}(i+1)}}\right) v_{\mathfrak{t s}_{i}}, \\
v_{\mathfrak{t}} X_{j} & =q^{\operatorname{res}_{\mathfrak{t}}(j)} v_{\mathfrak{t}}
\end{aligned}
$$

if $q \neq 1$; or

$$
\begin{aligned}
v_{\mathfrak{t}} s_{i} & =\frac{1}{\operatorname{res}_{\mathfrak{t}}(i+1)-\operatorname{res}_{\mathfrak{t}}(i)} v_{\mathfrak{t}}+\left(1+\frac{1}{\operatorname{res}_{\mathfrak{t}}(i+1)-\operatorname{res}_{\mathfrak{t}}(i)}\right) v_{\mathfrak{t}_{i}}, \\
v_{\mathfrak{t}} X_{j} & =\operatorname{res}_{\mathfrak{t}}(j) v_{\mathfrak{t}},
\end{aligned}
$$

if $q=1$, where if $\mathfrak{t}_{i}$ is not an $(l, m)$-special standard $\lambda / \mu$-tableau, then we define $v_{\mathrm{ts}_{i}}=0$. By Lemma 2.15, both (4.2) and (4.3) are well-defined.

Theorem 4.4 The above action extends to a well-defined action of $\mathcal{H}_{n}^{\text {aff }}$ on the $D(\lambda, \mu)$.

Proof. This can be verified by case by case arguments as in [R2].

Theorem 4.5 For each $(l, m)$-special skew shape $\nu=\lambda / \mu$ with $n$ nodes, $D(\lambda, \mu)$ is an irreducible $\mathcal{H}_{n}^{\text {aff }}$-module.

Lemma 4.6 Let $\lambda, \lambda^{\prime}$ be two $(l, m)$-special partitions of $n$ (with respect to $\emptyset$ ), $\mathfrak{s}$ (resp. $\mathfrak{t}$ ) be an $(l, m)$-special standard $\lambda$-tableau (resp. $(l, m)$-special standard $\lambda^{\prime}$-tableau) (with respect to $\emptyset$ ). If $\operatorname{res}_{\mathfrak{s}}(k)=\operatorname{res}_{\mathfrak{t}}(k), \forall 1 \leq k \leq n$, then $\lambda=\lambda^{\prime}$ and $\mathfrak{s}=\mathfrak{t}$.

Proof. We use induction on $n$. The case where $n=1$ is obvious. Suppose that the conclusion is true for $n-1$. We want to prove that it is also true for $n$. Let $\lambda, \lambda^{\prime}$ be two $(l, m)$-special partitions of $n$. Let $\mathfrak{s}$ (resp. $\left.\mathfrak{t}\right)$ be an $(l, m)$-special standard $\lambda$-tableau (resp. $(l, m)$-special standard $\lambda^{\prime}$-tableau) such that for any integer $k$ with $1 \leq k \leq n, \operatorname{res}_{\mathfrak{s}}(k)=\operatorname{res}_{\mathfrak{t}}(k)$. Let $\widetilde{\mathfrak{s}}:=\mathfrak{s} \downarrow_{n-1},[\widetilde{\lambda}]:=\operatorname{Shape}(\widetilde{\mathfrak{s}})$, $\widetilde{\mathfrak{t}}:=\mathfrak{t} \downarrow_{n-1},\left[\widetilde{\lambda}^{\prime}\right]:=$ Shape $(\widetilde{\mathfrak{t}})$. By induction hypothesis, we know that $\widetilde{\lambda}=\widetilde{\lambda}^{\prime}$ and $\widetilde{\mathfrak{s}}=\widetilde{\mathfrak{t}}$.

Let $\gamma$ be the node which is occupied by $n$ in $\mathfrak{s}$, and $\gamma^{\prime}$ be the node which is occupied by $n$ in $\mathfrak{t}$. Then both $\gamma, \gamma^{\prime}$ are addable nodes of $[\widetilde{\lambda}]$. It suffices to show that $\gamma=\gamma^{\prime}$. Suppose that $\gamma \neq \gamma^{\prime}$. Without loss of generality, we can assume that $\gamma$ is above $\gamma^{\prime}$. 
Case 1. both $\gamma$ and $\gamma^{\prime}$ are below the first row of $[\widetilde{\lambda}]$ and not below the last (nonzero) row of $[\widetilde{\lambda}]$.

Let $\gamma=(a, b), \gamma^{\prime}=\left(a^{\prime}, b^{\prime}\right)$, then $1 \leq a<a^{\prime} \leq \ell(\widetilde{\lambda}), \widetilde{\lambda}_{\ell(\widetilde{\lambda})} \leq b^{\prime}<b \leq \widetilde{\lambda}_{1}$. Since $\widetilde{\lambda}$ is $(l, m)$-special, it follows that $\ell(\widetilde{\lambda}) \leq m$ and $\widetilde{\lambda}_{1}-\widetilde{\lambda}_{\ell(\widetilde{\lambda})} \leq l-\ell(\widetilde{\lambda})$. Hence

$$
\begin{aligned}
0<\operatorname{Res}(\gamma)-\operatorname{Res}\left(\gamma^{\prime}\right) & =(b-a)-\left(b^{\prime}-a^{\prime}\right) \\
& \leq \widetilde{\lambda}_{1}-\widetilde{\lambda}_{\ell(\widetilde{\lambda})}+\ell(\widetilde{\lambda})-1 \leq l-1,
\end{aligned}
$$

which implies that $\operatorname{res}(\gamma) \neq \operatorname{res}\left(\gamma^{\prime}\right)$, a contradiction.

Case 2. $\gamma$ is in the first row of $[\widetilde{\lambda}]$, while $\gamma^{\prime}$ is below the first row of $[\widetilde{\lambda}]$ and not below the last (nonzero) row of $[\widetilde{\lambda}]$. Then $\gamma=\left(1, \widetilde{\lambda}_{1}+1\right), \gamma^{\prime}=\left(a^{\prime}, b^{\prime}\right)$, where

$$
1 \leq a^{\prime} \leq \ell(\widetilde{\lambda}), \quad \tilde{\lambda}_{\ell(\widetilde{\lambda})} \leq b^{\prime} \leq \tilde{\lambda}_{1} .
$$

Since $\lambda$ is $(l, m)$-special, it follows that $\ell(\widetilde{\lambda}) \leq m$ and $\widetilde{\lambda}_{1}+1-\widetilde{\lambda}_{m} \leq l-m$. Hence

$$
\begin{aligned}
0 & <\operatorname{Res}(\gamma)-\operatorname{Res}\left(\gamma^{\prime}\right)=\left(\widetilde{\lambda}_{1}+1-1\right)-\left(b^{\prime}-a^{\prime}\right) \\
& \leq l-m-1+\widetilde{\lambda}_{m}-\widetilde{\lambda}_{\ell(\widetilde{\lambda})}+\ell(\widetilde{\lambda}) \leq l-1,
\end{aligned}
$$

which implies that $\operatorname{res}(\gamma) \neq \operatorname{res}\left(\gamma^{\prime}\right)$, a contradiction.

Case 3. $\gamma$ is in the first row of $[\widetilde{\lambda}]$, while $\gamma^{\prime}$ is in the $(\ell(\widetilde{\lambda})+1)$-th row of $[\widetilde{\lambda}]$. Then $\gamma=\left(1, \widetilde{\lambda}_{1}+1\right), \gamma^{\prime}=(\ell(\widetilde{\lambda})+1,1)$.

Since $\lambda^{\prime}$ is $(l, m)$-special, it follows that $\ell(\widetilde{\lambda})+1 \leq m$. In particular $\ell(\widetilde{\lambda})<m$, hence $\widetilde{\lambda}_{m}=0$. Since $\lambda$ is $(l, m)$-special, it follows that $\widetilde{\lambda}_{1}+1 \leq l-m$. Hence

$$
\begin{aligned}
0 & <\operatorname{Res}(\gamma)-\operatorname{Res}\left(\gamma^{\prime}\right)=\left(\widetilde{\lambda}_{1}+1-1\right)-(1-\ell(\widetilde{\lambda})-1) \\
& \leq l-m-1+\ell(\widetilde{\lambda})<l-1,
\end{aligned}
$$

which implies that $\operatorname{res}(\gamma) \neq \operatorname{res}\left(\gamma^{\prime}\right)$, a contradiction.

Case 4. $\quad \gamma$ is below the first row of $[\widetilde{\lambda}]$ and not below the last (nonzero) row of $[\widetilde{\lambda}]$, while $\gamma^{\prime}$ is in the $(\ell(\widetilde{\lambda})+1)$-th row of $[\widetilde{\lambda}]$. Then $\gamma=(a, b), \gamma^{\prime}=(\ell(\widetilde{\lambda})+1,1)$, where

$$
1 \leq a \leq \ell(\widetilde{\lambda}), \quad \widetilde{\lambda}_{\ell(\widetilde{\lambda})} \leq b \leq \widetilde{\lambda}_{1}
$$

Since $\lambda^{\prime}$ is $(l, m)$-special, it follows that $\ell(\widetilde{\lambda})+1 \leq m$ and $\widetilde{\lambda}_{1}-1 \leq l-m$. Hence

$$
\begin{aligned}
0 & <\operatorname{Res}(\gamma)-\operatorname{Res}\left(\gamma^{\prime}\right)=(b-a)-(1-\ell(\widetilde{\lambda})-1) \\
& \leq \widetilde{\lambda}_{1}-1+\ell(\widetilde{\lambda}) \leq l-m+m-1=l-1,
\end{aligned}
$$


which implies that $\operatorname{res}(\gamma) \neq \operatorname{res}\left(\gamma^{\prime}\right)$, a contradiction. This completes the proof of the lemma.

Corollary 4.7 Let $\lambda / \mu$ and $\lambda^{\prime} / \mu$ be two $(l, m)$-special skew shapes (with respect to $\mu$ ), where $\mu \vdash k$ and $\lambda, \lambda^{\prime} \vdash(n+k)$ for some positive integers $k, n$. Let $\mathfrak{s}($ resp. $\mathfrak{t}$ ) be an $(l, m)$-special standard $\lambda / \mu$-tableau (resp. $(l, m)$-special standard $\lambda^{\prime} / \mu$-tableau) (with respect to $\mu$ ). If $\operatorname{res}_{\mathfrak{s}}(k)=\operatorname{res}_{\mathfrak{t}}(k), \forall 1 \leq k \leq n$, then $\lambda=\lambda^{\prime}$ and $\mathfrak{s}=\mathfrak{t}$.

Proof. Let $\mathfrak{t}_{\mu}$ be the standard $\mu$-tableau in which the number $-k, \cdots,-2,-1$ appear in order (from top to bottom) along successive columns. We define $\widehat{\mathfrak{s}}:=$ $\mathfrak{t}_{\mu} \cup \mathfrak{s}, \widehat{\mathfrak{t}}:=\mathfrak{t}_{\mu} \cup \mathfrak{t}$. It is clear that both $\widehat{\mathfrak{s}}$ and $\widehat{\mathfrak{t}}$ are $(l, m)$-special standard tableau with respect to $\mu$. Now applying Lemma 4.6, we get our corollary.

Theorem 4.8 Let $\lambda / \mu$ and $\lambda^{\prime} / \mu$ be two $(l, m)$-special skew shapes (with respect to $\mu$ ). Suppose that $\lambda \neq \lambda^{\prime}$, where $\mu \vdash k$ and $\lambda, \lambda^{\prime} \vdash(n+k)$ for some positive integers $k, n$. then $D(\lambda, \mu) \nRightarrow D\left(\lambda^{\prime}, \mu\right)$ as $\mathcal{H}_{n}^{\text {aff }}$-modules.

Proof. For each $(l, m)$-special standard $\lambda / \mu$-tableau $\mathfrak{t}$, we define

$$
\widetilde{\Theta}_{\mathfrak{t}}:=\prod_{\substack{\mu \subseteq \lambda \vdash n+k \\ \lambda \text { is }(l, m) \text {-special }}} \prod_{i=1}^{n} \prod_{\substack{\mathfrak{u} \in \operatorname{Std}_{l, m}(\lambda, \mu) \\ \operatorname{res}_{\mathfrak{u}}(i) \neq \operatorname{res}_{\mathfrak{t}}(i)}} \frac{X_{i}-q^{\operatorname{res}_{\mathfrak{u}}(i)}}{q^{\operatorname{res}_{\mathfrak{t}}(i)}-q^{\operatorname{res}_{\mathfrak{u}}(i)}},
$$

if $q \neq 1$; or

$$
\widetilde{\Theta}_{\mathfrak{t}}:=\prod_{\substack{\mu \subseteq \lambda \vdash n+k \\ \lambda \text { is }(l, m) \text {-special }}} \prod_{i=1}^{n} \prod_{\substack{\mathfrak{u} \in \operatorname{Std}_{l, m}(\lambda, \mu) \\ \operatorname{res}_{\mathfrak{u}}(i) \neq \operatorname{res}_{\mathfrak{t}}(i)}} \frac{X_{i}-\operatorname{res}_{\mathfrak{u}}(i)}{\operatorname{res}_{\mathfrak{t}}(i)-\operatorname{res}_{\mathfrak{u}}(i)},
$$

if $q=1$.

Now suppose that $\lambda / \mu, \lambda^{\prime} / \mu$ are two $(l, m)$-special skew shapes (with respect to $\mu$ ) such that there is a $\mathcal{H}_{n}^{\text {aff }}$-module isomorphism $\varphi: D(\lambda, \mu) \cong D\left(\lambda^{\prime}, \mu\right)$. Let $\mathfrak{t}$ be an $(l, m)$-special standard $\lambda / \mu$-tableau, and write

$$
\varphi\left(v_{\mathfrak{t}}\right)=\sum_{\mathfrak{u} \in \operatorname{Std}_{l, m}\left(\lambda^{\prime}, \mu\right)} a_{\mathfrak{u}} v_{\mathfrak{u}}
$$

By Corollary 4.7, for any two $(l, m)$-special standard tableaux (with respect to $\mu) \mathfrak{u}$ and $\mathfrak{v}, v_{\mathfrak{v}} \widetilde{\Theta}_{\mathfrak{u}}=\delta_{\mathfrak{u}, \mathfrak{v}} v_{\mathfrak{v}}$. Since $\varphi$ is a $\mathcal{H}_{n}^{\text {aff }}$-module homomorphism, we have $\varphi\left(v_{\mathfrak{t}}\right)=\varphi\left(v_{\mathfrak{t}} \widetilde{\Theta}_{\mathfrak{t}}\right)=\varphi\left(v_{\mathfrak{t}}\right) \widetilde{\Theta}_{\mathfrak{t}}$. From this and Corollary 4.7 it is easy to see that $\lambda=\lambda^{\prime}$, as required. 


\section{Identifying $D(\lambda)$ with $D^{\lambda}$}

Let $K, q, l, m$ be defined as before. Let $n$ be a positive integer. A composition of $n$ is a sequence $\mu=\left(\mu_{1}, \cdots, \mu_{k}\right)$ of non-negative integers such that $n=|\mu|:=$ $\sum_{i=1}^{k} \mu_{i}$. Let $\mu$ be a composition of $n$, then we have an obvious embedding

$$
\mathcal{H}_{\mu}^{\mathrm{aff}}:=\mathcal{H}_{\mu_{1}}^{\mathrm{aff}} \otimes \cdots \otimes \mathcal{H}_{\mu_{k}}^{\mathrm{aff}} \hookrightarrow \mathcal{H}_{n}^{\mathrm{aff}} .
$$

We call $\mathcal{H}_{\mu}^{\text {aff }}$ a Young subalgebra of $\mathcal{H}_{n}^{\text {aff }}$. Following [K2], we give

Definition 5.1 An irreducible $\mathcal{H}_{n}^{\text {aff }}$-module $D$ is called completely splittable if and only if the restriction $D \downarrow_{\mathcal{H}_{\mu}^{\text {aff }}}$ to any Young subalgebra $\mathcal{H}_{\mu}^{\text {aff }} \subseteq \mathcal{H}_{n}^{\text {aff }}$ is semi-simple.

Theorem 5.2 For any $(l, m)$-special skew shape $\lambda / \mu$, the irreducible representation $D(\lambda, \mu)$ is completely splittable.

Proof. This follows directly from our construction of $D(\lambda, \mu)$. that

Let $\nu=\lambda / \mu$ be an $(l, m)$-special skew shape with $n$ nodes. It is easy to see

$$
D(\lambda, \mu) \downarrow_{\mathcal{H}_{n-1}^{\text {aff }}} \cong \underset{\substack{\rho \rightarrow \nu \\ \rho=\lambda^{\prime} / \mu, \lambda^{\prime} \subset \lambda \\(1, \mathrm{~m}) \text {-special with respect to } \mu}}{\bigoplus} D\left(\lambda^{\prime}, \mu\right),
$$

where $\rho \rightarrow \nu$ means that $[\rho]$ is obtained from $[\nu]$ by removing a removable node in $[\nu]$.

Let $\mathcal{H}_{q}\left(\mathfrak{S}_{n}\right)$ be the Hecke algebra of $\mathfrak{S}_{n}$. Let $w \in \mathfrak{S}_{n}$. Given a reduced expression $s_{i_{1}} \cdots s_{i_{k}}$ for $w$, we write $T_{w}=T_{i_{1}} \cdots T_{i_{k}}$. The braid relations ensure that $T_{w}$ is independent of the choice of reduced expression. For any partition $\lambda$ of $n$, we denote by $\mathfrak{t}^{\lambda}$ (resp. $\mathfrak{t}_{\lambda}$ ) the standard $\lambda$-tableau in which the numbers $1,2, \cdots, n$ appear in order along successive rows (resp. columns). Let $w_{\lambda} \in \mathfrak{S}_{n}$ be such that $\mathfrak{t}^{\lambda} w_{\lambda}=\mathfrak{t}_{\lambda}$. Following [DJ1], we define

$$
x_{\lambda}:=\sum_{w \in \mathfrak{S}_{n}} T_{w}, \quad y_{\lambda}:=\sum_{w \in \mathfrak{S}_{n}}(-q)^{-\ell(w)} T_{w}, \quad z_{\lambda}:=x_{\lambda} T_{w_{\lambda}} y_{\lambda^{t}},
$$

where $\lambda^{t}$ is the conjugate partition of $\lambda$. Let $S^{\lambda}$ be the Specht module (see [DJ1]) for $\mathcal{H}_{q}\left(\mathfrak{S}_{n}\right)$. By definition, $S^{\lambda}=z_{\lambda} \mathcal{H}_{q}\left(\mathfrak{S}_{n}\right)$.

It is well-known that the map which sends (for each $1 \leq i<n$ ) $T_{i}$ to $T_{i}$, and sends $X_{1}$ to 1 if $q \neq 1$; or sends $X_{1}$ to 0 if $q=1$, extends naturally to a surjective algebra homomorphism from $\mathcal{H}_{n}^{\text {aff }}$ to $\mathcal{H}_{q}\left(\mathfrak{S}_{n}\right)$. In this way, any irreducible module over $\mathcal{H}_{q}\left(\mathfrak{S}_{n}\right)$ can be naturally regarded as an irreducible module over $\mathcal{H}_{n}^{\text {aff }}$.

Let $\lambda$ be an $l$-restricted partition. Then $D^{\lambda}:=S^{\lambda} / \operatorname{rad} S^{\lambda} \neq 0$. By $[\mathrm{K} 2], D^{\lambda}$ is completely splittable if and only if $\lambda$ is $(l, m)$-special for some given positive 
integer $m \leq l$. Note also that an $(l, m)$-special partition is automatically an $l$-restricted partition.

Let $M$ be a finite dimensional $\mathcal{H}_{n}^{\text {aff }}$-module. Following [Gr] (see also [K1] and $[\mathrm{V}]$ ), for each integer $i$ with $1 \leq i \leq n$, we define $e_{i} M$ to be the generalized eigenspace of $X_{n}-q^{i}$ in $M$ if $q \neq 1$; or the generalized eigenspace of $X_{n}-i$ in $M$ if $q=1$. We regard $e_{i} M$ as a module over $\mathcal{H}_{n-1}^{\text {aff }}$. For any $l$-restricted partition $\lambda$ of $n$, by [K1, (0.5)], [B, (2.6)] and [LLT], we have

$$
D^{\lambda} \downarrow_{\mathcal{H}_{n-1}^{\text {aff }}} \cong \bigoplus_{i=1}^{n} e_{i} D^{\lambda}, \quad \operatorname{soc}\left(e_{i} D^{\lambda}\right) \cong \bigoplus_{\substack{\mu \text { is an } l \text {-restricted } \\ \text { partition of } n-1, \mu \stackrel{i}{\rightarrow} \lambda}} D^{\mu},
$$

where $\mu \stackrel{i}{\rightarrow} \lambda$ means that $\mu$ is obtained from $\lambda$ by removing a removable node $\gamma$ with $\operatorname{res}(\gamma)=i$.

Now let $\lambda$ be an $(l, m)$-special partition. Then $\lambda$ is an $l$-restricted partition, and both $D(\lambda)$ and $D^{\lambda}$ are completely splittable. In particular, for any $1 \leq i \leq n$,

$$
\operatorname{soc}\left(e_{i} D(\lambda)\right)=e_{i} D(\lambda), \quad \operatorname{soc}\left(e_{i} D^{\lambda}\right)=e_{i} D^{\lambda} .
$$

By Lemma 2.14, we know that there are no two different removable nodes of $[\lambda]$ which have the same res values. On the other hand, for any two irreducible $\mathcal{H}_{n}^{\text {aff }}$-modules $L, M$, if $\operatorname{soc}\left(e_{i} L\right) \cong \operatorname{soc}\left(e_{i} M\right) \neq 0$ for some $1 \leq i \leq n$, then (by [V, $5.8(3)]) L \cong M$. In view of this and the decomposition (5.3), (5.4), we get (by using induction on $n$ ) that

Theorem 5.5 For each $(l, m)$-special partition $\lambda$, we have that $D(\lambda) \cong D^{\lambda}$.

Note that for each $(l, m)$-special partition $\lambda, \mathfrak{t}_{\lambda}$ is an $(l, m)$-special standard tableau. We have

Corollary 5.6 For each $(l, m)$-special partition $\lambda$, the map $\varphi$ which sends $z_{\lambda}$ to $v_{\mathfrak{t}_{\lambda}}$ extends naturally to a $\mathcal{H}_{n}^{\text {aff }}$-module isomorphism $D^{\lambda} \cong D(\lambda)$.

Proof. For each integer $a$ with $1 \leq a \leq n$, we define

$$
\begin{gathered}
L_{a}:= \begin{cases}0, & \text { if } a=1, \\
q^{1-a} T_{(1, a)}+q^{2-a} T_{(2, a)}+\cdots+q^{-1} T_{(a-1, a)}, & \text { if } 2 \leq a \leq n,\end{cases} \\
\widetilde{L}_{a}:= \begin{cases}1, & \text { if } a=1, \\
q^{1-a} T_{a-1} \cdots T_{1} T_{1} \cdots T_{a-1}, & \text { if } 2 \leq a \leq n,\end{cases}
\end{gathered}
$$

It is well-known $([\mathrm{DJ} 3,(4.2)])$ that $\widetilde{L}_{a}=1+(q-1) L_{a}$. By [DJ2, (3.14)], $z_{\lambda} L_{a}=$ $\left(1+q+\cdots+q^{j-i-1}\right) z_{\lambda}$, if $a$ is in position $(i, j)$ in $\mathfrak{t}_{\lambda}$. Hence $z_{\lambda} \widetilde{L}_{a}=q^{j-i} z_{\lambda}$, if $a$ is in position $(i, j)$ in $\mathfrak{t}_{\lambda}$. 
Let $\varphi: D^{\lambda} \cong D(\lambda)$ be the isomorphism given by Theorem 5.5. Write

$$
\varphi\left(z_{\lambda}\right)=\sum_{\mathfrak{u} \in \operatorname{Std}_{l, m}(\lambda, \emptyset)} a_{\mathfrak{u}} v_{\mathfrak{u}}
$$

By the construction of the surjective homomorphism from $\mathcal{H}_{n}^{\text {aff }}$ to $\mathcal{H}_{q}\left(\mathfrak{S}_{n}\right)$, it is easy to see that (for each $1 \leq a \leq n$ )

$$
z_{\lambda} X_{a}=q^{\text {rest }_{\lambda}(a)} z_{\lambda}
$$

if $q \neq 1$; or

if $q=1$.

$$
z_{\lambda} X_{a}=\operatorname{res}_{\mathfrak{t}_{\lambda}}(a) z_{\lambda}
$$

Now recall the definition of the operator $\widetilde{\Theta}_{\mathfrak{t}}$ in (4.9) and (4.10). Using the fact that $\varphi\left(z_{\lambda} \widetilde{\Theta}_{\mathfrak{t}_{\lambda}}\right)=\varphi\left(z_{\lambda}\right) \widetilde{\Theta}_{\mathfrak{t}_{\lambda}}$, and applying Lemma 4.6 , it is easy to see that $a_{\mathfrak{u}} \neq 0$ only if $\mathfrak{u}=\mathfrak{t}_{\lambda}$, as required.

\section{REFERENCES}

[A] S. Ariki, On the decomposition numbers of the Hecke algebra of $G(m, 1, n)$, J. Math. Kyoto Univ. 36 (1996), 789-808.

[AK] S. Ariki and K. Koike, A Hecke algebra of $(\mathbb{Z} / r \mathbb{Z}) \imath \mathfrak{S}_{n}$ and construction of its representations, Adv. Math. 106 (1994) 216-243.

[B] J. Brundan, Modular branching rules and the Mullineux map for Hecke algebras of type $A$, Proc. London. Math. Soc. (3) $\mathbf{7 7}$ (1998), 551-581.

[DJ1] R. Dipper and G. D. James, Representations of Hecke algebras of general linear groups, Proc. London. Math. Soc. (3) 52 (1986), 20-52.

[DJ2] R. Dipper and G. D. James, Blocks and idempotents of Hecke algebras of general linear groups, Proc. London. Math. Soc. (3) 54 (1987), 57-82.

[DJ3] R. Dipper and G. D. James, Representations of Hecke algebras of type $B_{n}, J$. Alg. 146 (1992), 454-481.

[Gr] I. Grojnowski, Affine $\widehat{\mathfrak{s l}}_{\ell}$ controls the modular representation theory of the symmetric group and related Hecke algebras, preprints.

[HaR] T. Halverson and A. Ram, Murnaghan-Nakayama rules characters of Iwahori-Hecke algebras of the complex reflection groups $G(r, p, n)$, Can. J. Math. (1) 50 (1997), 167-192.

[Ho] P.N. Hoefsmit, Representations of Hecke algebras of finite groups with $B N$-pairs of classical type, PhD thesis, University of British Columbia, 1974.

[Hu1] J. Hu, A Morita equivalence theorem for Hecke algebras of type $D_{n}$ when $n$ is even, Manuscripta Math. 108 (2002), 409-430.

[Hu2] J. Hu, Modular representations of Hecke algebras of type $G(p, p, n)$, J. Alg. (2) 274 (2004), 446-490.

[J] G. D. James, The decomposition matrices of $\mathrm{GL}_{n}(q)$ for $n \leq 10$, Proc. London. Math. Soc. 60 (1990), 225-265.

[K1] A. Kleshchev, Branching rules for modular representations of symmetric groups, J. reine. angew. Math. 459 (1995), 163-212.

[K2] A. Kleshchev, Completely splittable representations of symmetric groups, J. Alg. 181 (1996), 584-592. 
[LLT] A. Lascoux, B. Leclerc and J.-Y. Thibon, Hecke algebras at roots of unity and crystal bases quantum affine algebras, Comm. math. Phys. 181 (1996), 205-263.

[M] O. Mathieu, On the dimension of some modular irreducible representations of the symmetric group, Lett. Math. Phys. (1) 38 (1996), 23-32.

[R1] A. Ram, Seminormal representations of Weyl groups and Iwahori-Hecke algebras, Proc. London. Math. Soc. (3) 75 (1997), 99-133.

[R2] A. Ram, Calibrated representations of affine Hecke algebras, preprint 1998.

[R3] A. Ram, Skew shape representations are irreducible, in: Combinatorial and Geometric representation theory, S.-J. Kang and K.-H. Lee eds., Contemp. Math. 325 Amer. Math. Soc. 2003, 161-189.

[R4] A. Ram, Affine Hecke algebras and generalized standard Young tableaux, J. Alg. 260 (2003), 367-415.

[W] H. Wenzl, Hecke algebras of type $A_{n}$ and subfactors, Invent. Math. (2) 92 (1988), 349-383.

[V] M. Vazirani, Irreducible modules over the affine Hecke algebra: a strong multiplicity one result, Ph. D. thesis, University of California at Berkeley, 1998.

\section{Jun $\mathrm{Hu}$}

Department of Applied Mathematics

Beijing Institute of Technology

Beijing, 100081, P. R. China

E-mail: junhu303@yahoo.com.cn 